\title{
EXISTENCE THEOREM FOR NONLINEAR MICROPOLAR ELASTICITY*
}

\author{
Josip TAMBAČA ${ }^{1}$ AND IgOR VELČIĆ ${ }^{1}$
}

\begin{abstract}
In this paper we give an existence theorem for the equilibrium problem for nonlinear micropolar elastic body. We consider the problem in its minimization formulation and apply the direct methods of the calculus of variations. As the main step towards the existence theorem, under some conditions, we prove the equivalence of the sequential weak lower semicontinuity of the total energy and the quasiconvexity, in some variables, of the stored energy function.
\end{abstract}

Mathematics Subject Classification. 74A35, 74G25, 74G65.

Received April 7, 2008. Revised July 14, 2008.

Published online October 21, 2008.

\section{INTRODUCTION}

In this paper we investigate the existence of solutions of the equilibrium problem of nonlinear three-dimensional micropolar elasticity. Micropolar continuum is a generalized continuum for which, in contrast to the classical elasticity, the unknowns in the problem are the deformation field $\varphi$ and independent microrotation field $\overline{\mathbf{R}}$ (a function with values in rotations); namely the points are allowed to rotate without stretch. Such generalized continua are introduced by the Cosserat brothers in [5]. For the overview of the micropolar elasticity see [7]. For the physical relevance of the micropolar (and micromorphic) elasticity in conjunction with finite elasto-plasticity and elastic metallic foams see [18,21].

Existence theorems in the linearized micropolar elasticity are usually based on the uniform positivity of the stored energy function (see [9] or [1]). A new approach has been taken by Neff in [10,16] which avoids some inherent problems when relating the model to specific physical situations. The first existence theorems for geometrically exact Cosserat and micromorphic models, based on convexity arguments are given in [17] (micromorphic elasticity is more general theory than micropolar elasticity). Also, for generalized continua with microstructure the existence theorem is given in [11] where convexity in the derivative of the variable which describes microstructure is demanded (in the micropolar case that would mean convexity in $\nabla \overline{\mathbf{R}}$ ). In our work we extend these developments in the micropolar case to more general constitutive behavior.

The methods we apply are the direct methods of the calculus of variations. Therefore we consider the equilibrium problem of the micropolar elasticity as the minimization problem for the total energy functional and look for its minimizers. We restrict ourselves to the case of the stored density function satisfying the standard growth conditions of order $p$ as used in most works of classical finite elasticity. These conditions exclude to

Keywords and phrases. Micropolar elasticity, existence theorem, quasiconvexity.

* This work was supported in part by grant 037-0693014-2765 from the Ministry of Education, Science and Sports of the Republic of Croatia.

${ }^{1}$ Department of Mathematics, University of Zagreb, Bijenička 30, 10000 Zagreb, Croatia. tambaca@math.hr; ivelcic@math.hr 
describe the failure of materials. We adapt methods from $[3,6]$ applied in the case of classical elasticity. In the classical elasticity under some conditions on the stored density function the sequential weak lower semicontinuity of the total energy is equivalent to the quasiconvexity of the stored energy function in variable $\nabla \varphi$. We extend this result and give the necessary and sufficient condition on the stored energy function of the micropolar body such that the total energy functional is sequentially weakly lower semicontinuous. This is important because the sequential weak lower semicontinuity together with coerciveness of the internal energy implies the existence of minimizers of the total energy. Moreover, the sequential weak lower semicontinuity plays the role in the justification of the lower dimensional models from three-dimensional theories by means of $\Gamma$-convergence (for the derivation and justification of the models for geometrically exact Cosserat plates and shells see [15,19]). In micropolar theory the stored energy function should be quasiconvex in variable $\nabla \varphi$ and variable $\boldsymbol{\omega}$ which we introduce and which describes the derivatives of the micropolar rotation $\overline{\mathbf{R}}$. The main difficulty of the problem is that we are dealing with the nonlinear manifold $\mathrm{SO}(3)$. On the other hand the main drawback of the technique is that the condition $p>3$ on the space $W^{1, p}(\Omega)$, where we look for solution, is imposed. This excludes some interesting stored energy functions (like quadratic, which is analogous to the classical St. Venant Kirchhoff material, see $[4,25])$. We hope we will be able to overcome this condition.

By $\mathbf{A}_{\boldsymbol{v}}$ we denote skew-symmetric matrix associated to its axial vector $\boldsymbol{v}$, i.e. $\mathbf{A}_{\boldsymbol{v}} x=\boldsymbol{v} \times x$. Ву $\boldsymbol{a}_{\mathrm{T}}$ we denote the axial vector of $\mathbf{T}-\mathbf{T}^{T}$ i.e. $\mathbf{T}-\mathbf{T}^{T}=\mathbf{A}_{a_{\mathbf{T}}}$. Note that

$$
\mathbf{T} \cdot \mathbf{A}_{v}=a_{\mathbf{T}} \cdot v
$$

Summation convention for the repeated indices is used. I denotes a unit matrix in the appropriate dimension.

\section{Micropolar Elasticity}

Let $\Omega \subset \mathbb{R}^{m}$ be an open bounded set with Lipschitz boundary. We assume $\bar{\Omega}$ to be a reference configuration of a micropolar body. That means, in contrast to the classical elasticity where the motion of a material particle is fully described by a vector function called deformation function $\varphi: \Omega \rightarrow \mathbb{R}^{3}$, that material particles undergo an additional micromotion, corresponding to the rotation $\overline{\mathbf{R}}: \Omega \rightarrow \mathrm{SO}(3)$ of the material particle at the microscale. Micropolar continua is a special case of the microstretch continua, both introduced by A.C. Eringen in mid 1960s. For the foundation of the theory see [7].

As $\overline{\mathbf{R}}$ is a rotation there are vectors $\boldsymbol{\omega}_{i}$ such that

$$
\partial_{i} \overline{\mathbf{R}}=\boldsymbol{\omega}_{i} \times \overline{\mathbf{R}}, \quad i=1, \ldots, m,
$$

where the vector product is taken with respect to the columns of $\overline{\mathbf{R}}$. Vectors $\boldsymbol{\omega}_{i}$ can then be expressed in terms of $\overline{\mathbf{R}}=\left(\begin{array}{lll}\overline{\boldsymbol{R}}_{1} & \overline{\boldsymbol{R}}_{2} & \overline{\boldsymbol{R}_{3}}\end{array}\right)$ by

$$
\boldsymbol{\omega}_{i}=\frac{1}{2} \overline{\boldsymbol{R}}_{j} \times \partial_{i} \overline{\boldsymbol{R}}_{j}, \quad i=1, \ldots, m .
$$

The strain measures (deformation tensors) are given by

$$
\overline{\mathbf{U}}=\overline{\mathbf{R}}^{T} \nabla \varphi, \quad \boldsymbol{\Gamma}=\overline{\mathbf{R}}^{T} \boldsymbol{\omega},
$$

where $\boldsymbol{\omega}=\left(\begin{array}{lll}\boldsymbol{\omega}_{1} & \boldsymbol{\omega}_{2} & \boldsymbol{\omega}_{3}\end{array}\right)$; here $\overline{\mathbf{U}}$ is usually called the non-symmetric first Cosserat stretch tensor. Note here that for instance for $m=3$ in the rigid motion case $\overline{\mathbf{U}}=\mathbf{I}$ and $\boldsymbol{\Gamma}=0$. In the sequel we assume that the material is homogeneous and that the energy is a function of $\varphi, \overline{\mathbf{R}}, \boldsymbol{\omega}$ which is bounded below. To be more precise we shall assume that there exists a continuous stored energy function $W: \mathbb{R}^{3 \times m} \times \mathrm{SO}(3) \times \mathbb{R}^{3 \times m} \rightarrow \mathbb{R}$ (i.e. $W(\nabla \varphi, \overline{\mathbf{R}}, \boldsymbol{\omega})$ is the volume density of the internal energy of the body in the reference configuration). We shall not consider more realistic case when $W$ have singularities for $\operatorname{det} \nabla \varphi=0$. Our motivating examples for 
the stored energy function are polynomials in strains $\overline{\mathbf{U}}, \boldsymbol{\Gamma}$. The total energy is then given by

$$
I(\boldsymbol{\varphi}, \overline{\mathbf{R}})=\int_{\Omega}\left(W(\nabla \boldsymbol{\varphi}, \overline{\mathbf{R}}, \boldsymbol{\omega})-\Pi_{\boldsymbol{f}}(\boldsymbol{\varphi})-\Pi_{\mathbf{M}}(\overline{\mathbf{R}})\right) \mathrm{d} V-\int_{\Gamma_{S}} \Pi_{\boldsymbol{n}}(\boldsymbol{\varphi}) \mathrm{d} S-\int_{\Gamma_{C}} \Pi_{\mathbf{M}_{c}}(\overline{\mathbf{R}}) \mathrm{d} S .
$$

Let $\Gamma \subset \partial \Omega$ is a part of the boundary where the Dirichlet boundary conditions will be prescribed; $\Gamma_{S} \subset \partial \Omega$, $\Gamma \cap \Gamma_{S}=\emptyset$ is a part of the boundary, where traction boundary conditions in the form of the potential of applied surface forces $\Pi_{N}$ are given. In addition, $\Gamma_{C} \subset \partial \Omega, \Gamma \cap \Gamma_{C}=\emptyset$ is the part of the boundary where the potential of external surface couples are applied. On the remaining part of the boundary $\partial \Omega \backslash\left\{\Gamma \cup \Gamma_{S} \cup \Gamma_{C}\right\}$ the body is free of contact forces and couples. The potential of the external applied volume force is $\Pi_{\boldsymbol{f}}$ and $\Pi_{\mathbf{M}}$ takes the role of the potential of applied external volume couples. For simplicity we assume

$$
\Pi_{\boldsymbol{f}}(\boldsymbol{\varphi})=\langle\boldsymbol{f}, \boldsymbol{\varphi}\rangle, \Pi_{\mathbf{M}}(\overline{\mathbf{R}})=\langle\mathbf{M}, \overline{\mathbf{R}}\rangle, \Pi_{\boldsymbol{n}}(\boldsymbol{\varphi})=\langle\boldsymbol{n}, \boldsymbol{\varphi}\rangle, \Pi_{\mathbf{M}_{c}}(\overline{\mathbf{R}})=\left\langle\mathbf{M}_{c}, \overline{\mathbf{R}}\right\rangle
$$

for the potential of applied loads with given functions $f: \Omega \rightarrow \mathbb{R}^{3}, \mathbf{M}: \Omega \rightarrow \mathbb{R}^{3 \times 3}, \boldsymbol{n}: \Gamma_{S} \rightarrow \mathbb{R}^{3}, \mathbf{M}_{c}: \Gamma_{C} \rightarrow$ $\mathbb{R}^{3 \times 3}$ and where $\langle\cdot, \cdot\rangle$ stands for the standard scalar product.

Let the Dirichlet boundary conditions on $\Gamma$ are given by $\boldsymbol{g}_{d}: \Gamma \rightarrow \mathbb{R}^{3}, \overline{\mathbf{R}}_{d}: \Gamma \rightarrow \mathrm{SO}(3)$ and let

$$
\Phi=\left\{(\boldsymbol{\varphi}, \overline{\mathbf{R}}) \in W^{1, p}\left(\Omega, \mathbb{R}^{3}\right) \times W^{1, p}(\Omega, \operatorname{SO}(3)):\left.\varphi\right|_{\Gamma}=\boldsymbol{g}_{d},\left.\overline{\mathbf{R}}\right|_{\Gamma}=\overline{\mathbf{R}}_{d}\right\}
$$

where the boundary conditions are understood in the sense of traces and

$$
W^{1, p}(\Omega, \operatorname{SO}(3))=\left\{\overline{\mathbf{R}} \in W^{1, p}\left(\Omega, \mathbb{R}^{3 \times 3}\right): \overline{\mathbf{R}}(x) \in \mathrm{SO}(3) \text { for a.e. } x \in \Omega\right\}
$$

with the induced strong and weak topologies. Let us emphasize that the strong or the weak limit of the sequence of the elements of $W^{1, p}(\Omega, \mathrm{SO}(3))$ is also an element of that space. This is due to the fact that both convergences imply that $\overline{\mathbf{R}}_{k}(x) \rightarrow \overline{\mathbf{R}}(x)$ a.e. $x$ in $\Omega$ at least on a subsequence and this implies that $\overline{\mathbf{R}}(x)$ is a.e. a rotation. The similar statement was needed in [20].

Let $q$ be the conjugated exponent to $p$. We assume $\boldsymbol{f} \in L^{q}\left(\Omega, \mathbb{R}^{3}\right), \mathbf{M} \in L^{q}\left(\Omega, \mathbb{R}^{3 \times 3}\right), \boldsymbol{n} \in L^{q}\left(\Gamma_{S}, \mathbb{R}^{3}\right), \mathbf{M}_{c} \in$ $L^{q}\left(\Gamma_{C}, \mathbb{R}^{3 \times 3}\right), \boldsymbol{g}_{d} \in L^{p}\left(\Gamma, \mathbb{R}^{3}\right)$ and $\overline{\mathbf{R}}_{d}: \Gamma \rightarrow \mathrm{SO}(3)$ measurable. The equilibrium problem of the micropolar elastic body is now given by the minimization problem for the total energy functional $I$

$$
\text { find }(\varphi, \overline{\mathbf{R}}) \in \Phi, \quad I(\varphi, \overline{\mathbf{R}})=\inf _{(\boldsymbol{\psi}, \mathbf{S}) \in \Phi} I(\boldsymbol{\psi}, \mathbf{S})
$$

In the sequel we will study the minimizing sequence $\left(\varphi^{k}, \overline{\mathbf{R}}^{k}\right)_{k} \in \Phi$ of $I$, i.e.

$$
I\left(\varphi^{k}, \overline{\mathbf{R}}^{k}\right) \rightarrow \inf _{(\boldsymbol{\psi}, \mathbf{S}) \in \Phi} I(\boldsymbol{\psi}, \mathbf{S})
$$

Definition 2.1. Let $X$ be a Banach space and $I: X \rightarrow \mathbb{R}$. $I$ is sequentially weakly lower semicontinuous if for all $x \in X$ and all $\left(x_{k}\right)_{k} \subset X$ such that $x_{k} \rightarrow x$ weakly it follows

$$
I(x) \leq \liminf _{k \rightarrow \infty} I\left(x_{k}\right)
$$

The following proposition is well known in the classical elasticity.

Proposition 2.2. Let $\Omega \subset \mathbb{R}^{m}$ be an open bounded set with the Lipschitz boundary and $p \in\langle 1, \infty\rangle$. Let the minimizing sequence of the energy functional $I$ on $\Phi$ is bounded in $W^{1, p}\left(\Omega, \mathbb{R}^{3}\right) \times W^{1, p}(\Omega, \operatorname{SO}(3))$ and assume that $I$ is sequentially weakly lower semicontinuous in the same space. Then any weak limit of the minimizing sequence is a minimum point of $I$. 
Proof. Since $\left(\varphi^{k}, \overline{\mathbf{R}}^{k}\right)$ is bounded in $W^{1, p}\left(\Omega, \mathbb{R}^{3}\right) \times W^{1, p}(\Omega, \mathrm{SO}(3))$ it converges and there exist a weak limit $(\varphi, \overline{\mathbf{R}})$ at least for a subsequence. Note that $\overline{\mathbf{R}}$ is the element of $W^{1, p}(\Omega, \mathrm{SO}(3))$. Let

$$
m=\inf _{(\psi, \mathbf{S}) \in \Phi} I(\boldsymbol{\psi}, \mathbf{S}) .
$$

Note that compactness of the embeddings $W^{1, p}(\Omega) \hookrightarrow L^{p}(\Gamma)$ implies that $\left.\boldsymbol{\varphi}\right|_{\Gamma}=\boldsymbol{g}_{d},\left.\overline{\mathbf{R}}\right|_{\Gamma}=\overline{\mathbf{R}}_{d}$. Now we have

$$
m \leq I(\boldsymbol{\varphi}, \overline{\mathbf{R}}) \leq \liminf _{k \rightarrow \infty} I\left(\boldsymbol{\varphi}^{k}, \overline{\mathbf{R}}^{k}\right)=m
$$

which concludes the proof.

Remark 2.3. To guarantee the boundedness of the minimizing sequence one can, like in the classical elasticity, assume meas $(\Gamma)>0$ and the coerciveness of the stored energy function, i.e. that there exist constants $C_{1}>0$, $C_{2}$ such that

$$
W(\mathbf{A}, \overline{\mathbf{R}}, \mathbf{B}) \geq C_{1}\left(\|\mathbf{A}\|^{p}+\|\mathbf{B}\|^{p}\right)+C_{2}, \quad \forall \mathbf{A}, \mathbf{B} \in \mathbb{R}^{3 \times m}, \overline{\mathbf{R}} \in \mathrm{SO}(3) .
$$

Note that there are some physically significant situations where this is violated (see [18] how to deal with them).

Using the compactness of the embedding $W^{1, p}(\Omega) \hookrightarrow L^{p}(\Gamma)$ and $W^{1, p}(\Omega) \hookrightarrow L^{p}(\Omega)$ we see that the question of the sequentially weakly lower semicontinuity of the total energy functional reduces to the question of the sequentially weakly lower semicontinuity of the strain energy, i.e. of the functional (in the sequel denoted by $I$ )

$$
I(\boldsymbol{\varphi}, \overline{\mathbf{R}})=\int_{\Omega} W(\nabla \boldsymbol{\varphi}, \overline{\mathbf{R}}, \boldsymbol{\omega})
$$

Remark 2.4. The objectivity (frame-indifference) of the stored energy function implies

$$
W(\mathbf{Q A}, \mathbf{Q} \overline{\mathbf{R}}, \mathbf{Q B})=W(\mathbf{A}, \overline{\mathbf{R}}, \mathbf{B}), \quad \forall \mathbf{A}, \mathbf{B} \in \mathbb{R}^{3 \times m}, \overline{\mathbf{R}}, \mathbf{Q} \in \mathrm{SO}(3) .
$$

Now plugging in $\mathbf{Q}=\overline{\mathbf{R}}^{T}$ we conclude that there exists a function $\widetilde{W}: \mathbb{R}^{3 \times m} \times \mathbb{R}^{3 \times m} \rightarrow \mathbb{R}$ such that

$$
W(\mathbf{A}, \overline{\mathbf{R}}, \mathbf{B})=\widetilde{W}\left(\overline{\mathbf{R}}^{T} \mathbf{A}, \overline{\mathbf{R}}^{T} \mathbf{B}\right) .
$$

Therefore the stored energy function depends only on strains (this makes $\overline{\mathbf{U}}$ and $\boldsymbol{\Gamma}$ to be strain measures). The functions which satisfy (2.6) we call objective (frame-indifferent).

In the following two sections we will prove, under some conditions, the equivalence of the sequentially weakly lower semicontinuity and the quasiconvexity of the stored energy function with respect to the first and last variable. Therefore we have the following existence theorem.

Theorem 2.5. Let $\Omega \subset \mathbb{R}^{m}$ be an open bounded set with the Lipschitz boundary and $m<p<\infty$. Let $W: \mathbb{R}^{3 \times m} \times \mathrm{SO}(3) \times \mathbb{R}^{3 \times m} \rightarrow \mathbb{R}$ be a quasiconvex in the first and the last variable (see Thm. 3.9 for definition) and objective function which satisfies

(a) (growth condition) $W(\mathbf{A}, \overline{\mathbf{R}}, \mathbf{B}) \leq K\left(1+\|\mathbf{A}\|^{p}+\|\mathbf{B}\|^{p}\right), \quad \mathbf{A}, \mathbf{B} \in \mathbb{R}^{3 \times m}, \overline{\mathbf{R}} \in \mathrm{SO}(3)$,

(b) (coercivity) there exist $C_{1}>0$ and $C_{2} \in \mathbb{R}$ such that

$$
W(\mathbf{A}, \overline{\mathbf{R}}, \mathbf{B}) \geq C_{1}\left(\|\mathbf{A}\|^{p}+\|\mathbf{B}\|^{p}\right)+C_{2}, \quad \forall \mathbf{A}, \mathbf{B} \in \mathbb{R}^{3 \times m}, \overline{\mathbf{R}} \in \mathrm{SO}(3) .
$$

Then the total energy functional I given in (2.3) and (2.4) with meas $(\Gamma)>0$ attains its minimum in the set $\Phi$ if $\Phi$ is nonempty.

Proof. Directly from Proposition 2.2 and Corollary 4.11. 
Remark 2.6. Note that we have assumed that the material is homogeneous, i.e. the stored energy function $W$ is independent of the space variable $x$. We believe that nonhomogeneous case can be treated as well, by adapting the techniques from [6].

Remark 2.7. Let us consider the case when the coupling between $\nabla \boldsymbol{\varphi}$ and $\boldsymbol{\omega}$ is absent, i.e.

$$
W(\mathbf{A}, \overline{\mathbf{R}}, \mathbf{B})=W_{1}(\mathbf{A}, \overline{\mathbf{R}})+W_{2}(\overline{\mathbf{R}}, \mathbf{B})
$$

(this occurs for centrosymmetric bodies, see [23], p. 14). Let $W_{1}$ and $W_{2}$ be quasiconvex and objective and for $m<p<\infty$ and $1<r<\infty$ satisfy

(a) $W_{1}(\mathbf{A}, \overline{\mathbf{R}}) \leq K\left(1+\|\mathbf{A}\|^{r}\right), W_{2}(\overline{\mathbf{R}}, \mathbf{B}) \leq K\left(1+\|\mathbf{B}\|^{p}\right) \quad \mathbf{A}, \mathbf{B} \in \mathbb{R}^{3 \times m}, \overline{\mathbf{R}} \in \mathrm{SO}(3)$;

(b) there exist $C_{1}>0$ and $C_{2} \in \mathbb{R}$ such that

$$
W_{1}(\mathbf{A}, \overline{\mathbf{R}}) \geq C_{1}\|\mathbf{A}\|^{r}+C_{2}, W_{2}(\overline{\mathbf{R}}, \mathbf{B}) \geq C_{1}\|\mathbf{B}\|^{p}+C_{2} \quad \forall \mathbf{A}, \mathbf{B} \in \mathbb{R}^{3 \times m}, \overline{\mathbf{R}} \in \operatorname{SO}(3) .
$$

Then using the same techniques one can prove the existence of minimizers for the functional $I$ in the set

$$
\Phi=\left\{(\boldsymbol{\varphi}, \overline{\mathbf{R}}) \in W^{1, r}\left(\Omega, \mathbb{R}^{3}\right) \times W^{1, p}(\Omega, \operatorname{SO}(3)):\left.\varphi\right|_{\Gamma}=\mathbf{g}_{d},\left.\overline{\mathbf{R}}\right|_{\Gamma}=\overline{\mathbf{R}}_{d}\right\}
$$

provided that meas $(\Gamma)>0$.

Remark 2.8. If we introduce a simple isotropic quadratic stored energy function (as treated e.g. in [14]) of the type

$$
W(\nabla \boldsymbol{\varphi}, \overline{\mathbf{R}}, \boldsymbol{\omega})=\mu\|\operatorname{sym} \overline{\mathbf{U}}-\mathbf{I}\|^{2}+\mu_{c}\|\operatorname{skew} \overline{\mathbf{U}}\|^{2}+\frac{\lambda}{2} \operatorname{tr}[\overline{\mathbf{U}}-\mathbf{I}]^{2}+\mu L_{c}^{p}\|\boldsymbol{\omega}\|^{p}, p>3
$$

we conclude that the coerciveness assumption would imply $\mu_{c}>0$. This is undesirable property since there are some physical situations where $\mu_{c}=0$ is a reasonable choice (see [18]). However, in the existence proof the coerciveness is needed just to conclude that the minimizing sequence is bounded. Therefore we can deal with this situation like in [17], using extended three dimensional Korn's inequality proved in [24] (which improves the result in [13]). Also note that the existence result for this energy (which can be proved by convexity arguments, see [17]) is guaranteed by Remark 2.7.

Remark 2.9. Some nontrivial examples of stored energy functions covered by Theorem 2.5 can be found in the form

$$
W(\mathbf{A}, \overline{\mathbf{R}}, \mathbf{B})=W_{1}\left(\overline{\mathbf{R}}^{T} \mathbf{A}\right)+W_{2}\left(\overline{\mathbf{R}}^{T} \mathbf{B}\right)
$$

where $W_{1}, W_{2}$ are polyconvex functions (see [6], p. 99) that satisfy growth assumptions (a) and (b). As polyconvexity implies quasiconvexity (see [6], p. 102) the Theorem 2.5 can be applied in this case.

At the end of this section note that usually the stored energy function $W$ is chosen to depend on $\nabla \boldsymbol{\varphi}, \overline{\mathbf{R}}$ and $\partial_{i} \overline{\mathbf{R}}$. Instead of that we have assumed the dependence on $\nabla \boldsymbol{\varphi}, \overline{\mathbf{R}}$ and $\boldsymbol{\omega}$. Motivation for this change was that, due to $\overline{\mathbf{R}}$ being rotation (it belongs to the three-dimensional manifold $\mathrm{SO}(3)$ ), derivatives of $\overline{\mathbf{R}}$ are dependent (there are 27 of them). However, $\boldsymbol{\omega}$ has independent components and there is one-to-one, purely algebraic, correspondence between $(\overline{\mathbf{R}}, \partial \overline{\mathbf{R}})$ and $(\overline{\mathbf{R}}, \boldsymbol{\omega})$ for $\overline{\mathbf{R}}$ being a rotation. Note as well that there is an analogy between vector columns of $\boldsymbol{\omega}$ and angular velocity. For this change the Lemma 2.10 is essential. That all $27 \partial_{i} \overline{\mathbf{R}}$ derivatives can be controlled (and expressed) by 9 independent components is already noted in [22] where Curl $\overline{\mathbf{R}}$ is suggested as curvature measure. The reason why we work with $\boldsymbol{\omega}$ is the way the oscillations of $\overline{\mathbf{R}}$ affect $\boldsymbol{\omega}$ (see Lem. 3.7).

For $\overline{\mathbf{R}} \in W^{1, p}(\Omega, \mathrm{SO}(3))$ using (2.1) we associate the mapping $\overline{\mathbf{R}} \mapsto \boldsymbol{\omega}(\overline{\mathbf{R}}) \in W^{1, p}\left(\Omega, \mathbb{R}^{3 \times 3}\right)$ and by abuse of notation denote

$$
\boldsymbol{\omega}_{i}=\boldsymbol{\omega}(\overline{\mathbf{R}})_{i}=\frac{1}{2} \overline{\boldsymbol{R}}_{j} \times \partial_{i} \overline{\boldsymbol{R}}_{j}, \quad i=1, \ldots, m .
$$


Lemma 2.10. Let $\Omega \subset \mathbb{R}^{m}$ be a bounded open set and $p \in\langle 1, \infty]$. Let $\overline{\mathbf{R}}^{k}, \overline{\mathbf{R}} \in W^{1, p}(\Omega, \mathrm{SO}(3))$ and let $\boldsymbol{\omega}_{i}^{k}=\boldsymbol{\omega}^{k}\left(\overline{\mathbf{R}}^{k}\right)_{i}, \boldsymbol{\omega}_{i}=\boldsymbol{\omega}(\overline{\mathbf{R}})_{i}$. Then $\overline{\mathbf{R}}^{k} \rightarrow \overline{\mathbf{R}}$ in $W^{1, p}(\Omega, \operatorname{SO}(3))$ if and only if

$$
\overline{\mathbf{R}}^{k} \rightarrow \overline{\mathbf{R}} \text { in } L^{p}\left(\Omega, \mathbb{R}^{9}\right) \text { and } \boldsymbol{\omega}_{i}^{k} \rightarrow \boldsymbol{\omega}_{i} \text { in } L^{p}\left(\Omega, \mathbb{R}^{3}\right), i=1, \ldots, m
$$

Moreover, the same holds for the weak convergence (weak ${ }^{*}$ for $\left.p=\infty\right)$.

Proof. By approximating $\Omega$ by a increasing countable union of open sets which are finite union of open cubes and compactness of the embedding $W^{1, p}\left(\Omega^{\prime}\right) \hookrightarrow L^{p}\left(\Omega^{\prime}\right)$ for $\Omega^{\prime}$ bounded open set with Lipschitz boundary, we conclude that at least on a subsequence: $\overline{\mathbf{R}}^{k}(x) \rightarrow \overline{\mathbf{R}}(x)$ for a.e. $x \in \Omega$. Since $\boldsymbol{\omega}_{i}^{k}$ is given by terms $\partial_{i} \overline{\mathbf{R}}_{j l}^{k} \overline{\mathbf{R}}_{m n}^{k}$ it is enough to prove that if $f^{k} \rightarrow f\left(f^{k} \rightarrow f\right)$ in $L^{p}(\Omega)$ and $g^{k} \rightarrow g$ a.e. and if there exists $M$ such that $\left\|g^{k}\right\|_{L^{\infty}(\Omega)} \leq M$ then $f^{k} g^{k} \rightarrow f g\left(f^{k} g^{k} \rightarrow f g\right)$ in $L^{p}(\Omega)$. Let us consider the strong convergence first

$$
\int_{\Omega}\left|f^{k} g^{k}-f g\right|^{p} \leq 2^{p-1}\left(\int_{\Omega}\left|f^{k}-f\right|^{p}\left|g^{k}\right|^{p}+\int_{\Omega}|f|^{p}\left|g^{k}-g\right|^{p}\right) .
$$

The first term on the right hand side tends to zero as $f_{k} \rightarrow f$ in $L^{p}(\Omega)$ and $\left\|g^{k}\right\|_{L^{\infty}(\Omega)} \leq M$. The second term on the right hand side tends to zero by the Lebesgue dominated convergence theorem.

For the weak convergence since $f^{k} g^{k}$ is bounded in $L^{p}(\Omega)$ it is enough to use test functions $\psi \in L^{\infty}(\Omega)$. We have to prove that $\int_{\Omega} f^{g} g^{k} \psi \rightarrow \int_{\Omega} f g \psi$. Similarly as before one has

$$
\int_{\Omega}\left(f^{k} g^{k} \psi-f g \psi\right)=\int_{\Omega} f^{k}\left(g^{k}-g\right) \psi+\int_{\Omega}\left(f^{k}-f\right) g \psi .
$$

The first term on the right hand side tends to zero by the Hölder inequality and the Lebesgue dominated convergence theorem. The second term on the right hand side tends to zero by the property $f^{k} \rightarrow f$.

Since, by the same argumentation, every subsequence has its subsequence such that these convergences are satisfied, we have proved the theorem.

Remark 2.11. In the case $p=1$ from Dunford-Pettis theorem we conclude

$$
f^{k} \rightarrow f \text { in } L^{1} \text { and } g^{k} \rightarrow g \text { a.e. and }\left\|g^{k}\right\|_{L^{\infty}} \leq M \Longrightarrow f^{k} g^{k} \rightarrow f g \text { in } L^{1},
$$

which implies the statement of Lemma 2.10 for $p=1$ as well.

\section{NECESSITY OF QUASICONVEXITY}

In the sequel we shall show that quasiconvexity in the first and the last variable of the stored energy function is a necessary condition for the sequentially weakly lower semicontinuity of the functional $I$. We first recall the definition of quasiconvexity and proceed with a few technical lemmas.

Definition 3.1. The function $f: \mathbb{R}^{n \times m} \rightarrow \mathbb{R}$ is quasiconvex if

$$
f(\mathbf{A}) \leq \frac{1}{\operatorname{meas}(D)} \int_{D} f(\mathbf{A}+\nabla \chi(x)) \mathrm{d} x
$$

for every open bounded set $D \subset \mathbb{R}^{m}$ with Lipschitz boundary, for every $\mathbf{A} \in \mathbb{R}^{n \times m}$ and $\chi \in W_{0}^{1, \infty}\left(D, \mathbb{R}^{n}\right)$.

In the last definition $W_{0}^{1, \infty}\left(D, \mathbb{R}^{n}\right)$ is understood in the sense of Meyers see [12] i.e. set of $W^{1, \infty}\left(D, \mathbb{R}^{n}\right)$ functions with the zero trace at the boundary; that is different from the closure of $C_{0}^{\infty}\left(D, \mathbb{R}^{n}\right)$ in $W^{1, \infty}\left(D, \mathbb{R}^{n}\right)$ norm. 
One should also note that in the definition of quasiconvexity it is enough to demand the property for an arbitrary cube $D$ (see Dacorogna [6], Rem. viii, p. 101).

The following two lemmas are just the applications of Nemytsky operators, see [2], p. 15.

Lemma 3.2. Let $\Omega \subset \mathbb{R}^{m}$ be a bounded set and $p \in[1, \infty\rangle$. Let $f: \mathbb{R}^{m} \rightarrow \mathbb{R}$ be a continuous function that satisfies growth condition

$$
|f(\mathbf{A})| \leq K\left(1+\|\mathbf{A}\|^{p}\right) .
$$

Let $\boldsymbol{g}_{k}, \boldsymbol{g} \in L^{p}\left(\Omega ; \mathbb{R}^{m}\right)$ and $\boldsymbol{g}_{k} \rightarrow \boldsymbol{g}$ strongly in $L^{p}\left(\Omega ; \mathbb{R}^{m}\right)$. Then

$$
\int_{\Omega} f\left(\boldsymbol{g}_{k}\right) \rightarrow \int_{\Omega} f(\boldsymbol{g})
$$

Remark 3.3. For $p=\infty$ the Lemma holds with no growth condition on $f$.

Lemma 3.4. Let $f: \mathbb{R}^{n \times m} \rightarrow \mathbb{R}$ be continuous and satisfies the growth condition $|f(\mathbf{A})|<K\left(1+\|\mathbf{A}\|^{p}\right)$. Let $D$ be an open bounded set in $\mathbb{R}^{m}$ with Lipschitz boundary. Then we have

$$
\begin{aligned}
\frac{1}{\operatorname{meas}(D)} \inf _{\chi \in C_{0}^{\infty}\left(D, \mathbb{R}^{n}\right)} \int_{D} f(\mathbf{A}+\nabla \chi(x)) \mathrm{d} x & =\frac{1}{\operatorname{meas}(D)} \inf _{\chi \in W_{0}^{1, \infty}\left(D, \mathbb{R}^{n}\right)} \int_{D} f(\mathbf{A}+\nabla \chi(x)) \mathrm{d} x \\
& =\frac{1}{\operatorname{meas}(D)} \inf _{\chi \in W_{0}^{1, p}\left(D, \mathbb{R}^{n}\right)} \int_{D} f(\mathbf{A}+\nabla \chi(x)) \mathrm{d} x
\end{aligned}
$$

Remark 3.5. The first equality holds if $f$ is just continuous, since for every $\chi \in W_{0}^{1, \infty}\left(D, \mathbb{R}^{3}\right)$ there exist a sequence $\left(\boldsymbol{\psi}_{k}\right)_{k} \subset C_{0}^{\infty}\left(D, \mathbb{R}^{n}\right)$ such that $\left\|\boldsymbol{\psi}_{k}-\chi\right\|_{L^{\infty}\left(\Omega, \mathbb{R}^{n}\right)} \rightarrow 0$ and $\left\|\nabla \boldsymbol{\psi}_{k}\right\| \leq M$ for some $M>0$ and $\nabla \boldsymbol{\psi}_{k}(x) \rightarrow \nabla \boldsymbol{\chi}(x)$ a.e. in $D$ (see Meyers [12]). To establish the first equality in Lemma 3.4 use just the continuity of $f$ and the Lebesgue dominated convergence theorem.

In order to attain the quasiconvexity of the stored energy function in the classical elasticity one needs to oscillate the deformation and to find the derivative of the oscillations. Here we need to oscillate the rotations as well. The following two lemmas are crucial for proving the necessity of the quasiconvexity (Thm. 3.9). Lemma 3.6 determines the essential part of the derivative of the particular oscillations of the identity rotation. It enables us to define the oscillations of an arbitrary rotation (in Lem. 3.7) and to derive that the derivatives of the oscillation of rotation (expressed by function $\boldsymbol{\omega}$ ) oscillate $\boldsymbol{\omega}$ in the similar way as the derivatives of oscillations of $\boldsymbol{\varphi}$ oscillate $\nabla \boldsymbol{\varphi}$. Recall that $\mathbf{A}_{\boldsymbol{b}}$ denotes the antisymmetric matrix with axial vector $\boldsymbol{b}$.

Lemma 3.6. Let $D$ be a cube in $\mathbb{R}^{m}$ and let $\boldsymbol{\psi} \in C_{0}^{\infty}\left(D, \mathbb{R}^{3}\right)$. We extend $\boldsymbol{\psi}$ by periodicity to $\mathbb{R}^{m}$ and define $\mathbf{O}^{\delta}(x)=\exp \left(\mathbf{A}_{\delta \boldsymbol{\psi}\left(\frac{x}{\delta}\right)}\right)$ for $0<\delta<1$. Then there exist a constant $K$ independently of $\boldsymbol{\psi}$ and $\delta$ such that

$$
\begin{aligned}
& \left\|\mathbf{O}^{\delta}(x)-\mathbf{I}\right\| \leq K \delta \exp M(\boldsymbol{\psi}), \quad x \in \Omega, \\
& \left\|\partial_{i} \mathbf{O}^{\delta}(x)-\mathbf{A}_{\partial_{i}} \boldsymbol{\psi}\left(\frac{x}{\delta}\right)\right\| \leq K \delta M(\boldsymbol{\psi})(\exp (M(\boldsymbol{\psi}))-1), \quad x \in \Omega ;
\end{aligned}
$$

here $M(\boldsymbol{\psi})=\|\boldsymbol{\psi}\|_{W^{1, \infty}\left(D ; \mathbb{R}^{3}\right)}$.

Proof. From the definition of the exponential function it follows

$$
\exp \left(\mathbf{A}_{\delta \boldsymbol{\psi}\left(\frac{x}{\delta}\right)}\right)-\mathbf{I}=\sum_{k=1}^{\infty} \frac{\delta^{k} \mathbf{A}_{\boldsymbol{\psi}\left(\frac{x}{\delta}\right)}^{k}}{k !}
$$

For the operator norm one has $\left\|\mathbf{A}_{b}\right\| \leq\|b\|$ and $\|\mathbf{A B}\| \leq\|\mathbf{A}\|\|\mathbf{B}\|$. Therefore the first inequality in the statement of the lemma follows. 
Differentiating $\mathbf{O}^{\delta}$ term by term we obtain

$$
\begin{aligned}
& \partial_{i} \mathbf{O}^{\delta}(x)=\mathbf{A}_{\partial_{i} \psi\left(\frac{x}{\delta}\right)}+\frac{\delta}{2 !}\left(\mathbf{A}_{\partial_{i} \boldsymbol{\psi}\left(\frac{x}{\delta}\right)} \mathbf{A}_{\boldsymbol{\psi}\left(\frac{x}{\delta}\right)}+\mathbf{A}_{\boldsymbol{\psi}\left(\frac{x}{\delta}\right)} \mathbf{A}_{\partial_{i} \boldsymbol{\psi}\left(\frac{x}{\delta}\right)}\right) \\
& +\frac{\delta^{2}}{3 !}\left(\mathbf{A}_{\boldsymbol{\psi}\left(\frac{x}{\delta}\right)}^{2} \mathbf{A}_{\partial_{i} \boldsymbol{\psi}\left(\frac{x}{\delta}\right)}+\mathbf{A}_{\boldsymbol{\psi}\left(\frac{x}{\delta}\right)} \mathbf{A}_{\partial_{i} \boldsymbol{\psi}\left(\frac{x}{\delta}\right)} \mathbf{A}_{\boldsymbol{\psi}\left(\frac{x}{\delta}\right)}+\mathbf{A}_{\partial_{i} \boldsymbol{\psi}\left(\frac{x}{\delta}\right)} \mathbf{A}_{\boldsymbol{\psi}\left(\frac{x}{\delta}\right)}^{2}\right)+\ldots
\end{aligned}
$$

Taking $\mathbf{A}_{\partial_{i} \psi\left(\frac{x}{\delta}\right)}$ on the left hand side of the equation and taking the operator norm implies the second inequality in the statement of the lemma.

The following lemma is crucial for the proving of the necessity of the quasiconvexity. It tells that the oscillations converge weakly in $W^{1, p}\left(\Omega ; \mathbb{R}^{m}\right)$.

Lemma 3.7. Let $D$ be a cube, $p \in\langle 1, \infty], \varphi \in W^{1, p}\left(D, \mathbb{R}^{3}\right)$ and $\overline{\mathbf{R}} \in W^{1, p}(D, \operatorname{SO}(3))$. Let us extend $\boldsymbol{\chi}, \boldsymbol{\psi} \in$ $C_{0}^{\infty}\left(D, \mathbb{R}^{3}\right)$ to $\mathbb{R}^{m}$ by periodicity and for $k \in \mathbb{N}$ define

$$
\varphi^{k}(x)=\varphi(x)+\frac{1}{k} \chi(k x), \quad \overline{\mathbf{R}}^{k}(x)=\exp \left(\mathbf{A}_{\frac{1}{k}} \boldsymbol{\psi}(k x)\right) \overline{\mathbf{R}}(x)
$$

Then $\varphi^{k}=\varphi, \overline{\mathbf{R}}^{k}=\overline{\mathbf{R}}$ on $\partial D$ and as $k \rightarrow \infty$ one has

$$
\varphi^{k} \rightarrow \varphi, \quad \overline{\mathbf{R}}^{k} \rightarrow \overline{\mathbf{R}}
$$

weakly in $W^{1, p}\left(D, \mathbb{R}^{3}\right)$ i.e. weakly in $W^{1, p}\left(D, \mathbb{R}^{3 \times 3}\right)$. Moreover, there exists a constant $K$ independent of $\boldsymbol{\psi}$ such that

$$
\begin{aligned}
& \left\|\boldsymbol{\omega}^{k}(x)-\boldsymbol{\omega}(x)-\nabla \boldsymbol{\psi}(n x)\right\| \leq K \frac{1}{k} \exp (M(\boldsymbol{\psi}))(M(\boldsymbol{\psi})+\|\boldsymbol{\omega}(x)\|) \\
& \left\|\overline{\mathbf{R}}^{k}(x)-\overline{\mathbf{R}}(x)\right\| \leq K \frac{1}{k} \exp M(\boldsymbol{\psi})
\end{aligned}
$$

for a.e. $x \in D$ where $M(\boldsymbol{\psi})=\|\boldsymbol{\psi}\|_{W_{0}^{1, \infty}\left(D ; \mathbb{R}^{3}\right)}$ and $\boldsymbol{\omega}^{k}=\boldsymbol{\omega}\left(\overline{\mathbf{R}}^{k}\right), \boldsymbol{\omega}=\boldsymbol{\omega}(\overline{\mathbf{R}})$.

Proof. It is obvious that $\varphi^{k}=\boldsymbol{\varphi}, \overline{\mathbf{R}}^{k}=\overline{\mathbf{R}}$ on $\partial D$ and that $\varphi^{k} \rightarrow \boldsymbol{\varphi}$ in $L^{\infty}\left(D, \mathbb{R}^{3}\right)$. From Lemma 3.6 it follows that $\overline{\mathbf{R}}^{k} \rightarrow \overline{\mathbf{R}}$ in $L^{\infty}\left(D, \mathbb{R}^{3 \times 3}\right)$. To prove the weak convergences for $p>1$ is now equivalent to prove the boundedness of the derivatives of $\varphi^{k}$ and $\overline{\mathbf{R}}^{k}$ in $L^{p}(\Omega)$. For $\varphi^{k}$ this is obvious since

$$
\nabla \varphi^{k}(x)=\nabla \varphi(x)+\nabla \chi(k x)
$$

For $\overline{\mathbf{R}}^{k}$ is enough to establish (3.1) since $\left\|\partial_{i} \overline{\mathbf{R}}^{k}\right\| \leq\left\|\boldsymbol{\omega}^{k}\right\|\left\|\overline{\mathbf{R}}^{k}\right\|$, so boundedness of $\boldsymbol{\omega}^{k}$ implies boundedness of $\partial_{i} \overline{\mathbf{R}}^{k}$. Let us calculate

$$
\begin{aligned}
\partial_{i} \overline{\mathbf{R}}^{k}(x) & =\partial_{i} \mathbf{O}_{k}(x) \overline{\mathbf{R}}(x)+\mathbf{O}_{k}(x) \partial_{i} \overline{\mathbf{R}}(x) \\
& =\left(\partial_{i} \mathbf{O}_{k}(x)-\mathbf{A}_{\partial_{i} \psi(k x)}\right) \overline{\mathbf{R}}(x)+\mathbf{A}_{\partial_{i} \psi(k x)} \overline{\mathbf{R}}(x)+\left(\mathbf{O}_{k}(x)-\mathbf{I}\right) \partial_{i} \overline{\mathbf{R}}(x)+\partial_{i} \overline{\mathbf{R}}(x) .
\end{aligned}
$$

Thus we have

$$
\begin{aligned}
\boldsymbol{\omega}_{i}^{k}(x)= & \boldsymbol{\omega}_{i}(x)+\partial_{i} \boldsymbol{\psi}(k x)+\frac{1}{2}\left(\overline{\boldsymbol{R}}_{j}^{k}(x)-\overline{\boldsymbol{R}}_{j}(x)\right) \times \partial_{i} \overline{\boldsymbol{R}}_{j}(x)+\frac{1}{2}\left(\overline{\boldsymbol{R}}_{j}^{k}(x)-\overline{\boldsymbol{R}}_{j}(x)\right) \times\left(\partial_{i} \boldsymbol{\psi}(k x) \times \overline{\boldsymbol{R}}_{j}(x)\right) \\
& +\frac{1}{2} \overline{\boldsymbol{R}}_{j}^{k}(x) \times\left[\left(\partial_{i} \mathbf{O}_{k}(x)-\mathbf{A}_{\partial_{i} \psi(k x)}\right) \overline{\boldsymbol{R}}_{j}(x)\right]+\frac{1}{2} \overline{\boldsymbol{R}}_{j}^{k}(x) \times\left[\left(\mathbf{O}_{k}(x)-I\right) \partial_{i} \overline{\boldsymbol{R}}_{j}(x)\right] .
\end{aligned}
$$


Now using Lemma 3.6 and $\left\|\partial_{i} \overline{\boldsymbol{R}}_{j}\right\| \leq C\left\|\boldsymbol{\omega}_{i}\right\|$, for some $C>0$, it follows (3.1). The estimate (3.2) is the direct consequence of Lemma 3.6.

Remark 3.8. The statement of the previous lemma also holds for $p=1$. For that we need Remark 2.11, the fact that $\nabla \boldsymbol{\chi}(k x) \rightarrow 0, \nabla \boldsymbol{\psi}(k x) \rightarrow 0$ in $L^{1}\left(D, \mathbb{R}^{3 \times 3}\right)$ and relation (3.1).

We are now ready for the main theorem. Since the weak $*$ convergence in $W^{1, \infty}\left(\Omega, \mathbb{R}^{m}\right)$ implies weak convergence in $W^{1, p}\left(\Omega, \mathbb{R}^{m}\right)$ we shall prove the necessity of quasiconvexity for sequentially weakly lower semicontinuity in spaces $W^{1, \infty}\left(\Omega, \mathbb{R}^{m}\right)$. We follow [6], p. 69 .

Theorem 3.9. Let $\Omega \subset \mathbb{R}^{m}$ be an open bounded set, let $f: \mathbb{R}^{3 \times m} \times \mathrm{SO}(3) \times \mathbb{R}^{3 \times m} \rightarrow \mathbb{R}$ be continuous and let the functional defined by

$$
I(\varphi, \overline{\mathbf{R}})=\int_{\Omega} f(\nabla \varphi(x), \overline{\mathbf{R}}(x), \boldsymbol{\omega}(x)) \mathrm{d} x
$$

be sequentially weakly lower semicontinuous, i.e. it satisfies the condition

$$
I(\boldsymbol{\varphi}, \overline{\mathbf{R}}) \leq \liminf _{k \rightarrow \infty} I\left(\varphi^{k}, \overline{\mathbf{R}}^{k}\right)
$$

for every sequence $\left(\left(\varphi^{k}, \overline{\mathbf{R}}^{k}\right)\right)_{k} \subset W^{1, \infty}\left(\Omega ; \mathbb{R}^{3}\right) \times W^{1, \infty}(\Omega ; \mathrm{SO}(3))$ that converges weak $*$ to $(\boldsymbol{\varphi}, \overline{\mathbf{R}})$ in $W^{1, \infty}\left(\Omega ; \mathbb{R}^{3}\right) \times W^{1, \infty}\left(\Omega ; \mathbb{R}^{3 \times 3}\right)$.

Then $f$ is quasiconvex in the first and the last variable i.e. $f$ satisfies

$$
f(\mathbf{A}, \overline{\mathbf{R}}, \mathbf{B}) \leq \frac{1}{\operatorname{meas}(D)} \int_{D} f(\mathbf{A}+\nabla \chi(x), \overline{\mathbf{R}}, \mathbf{B}+\nabla \boldsymbol{\psi}(x)) \mathrm{d} x
$$

for every open bounded set $D$ with Lipschitz boundary, for every $\mathbf{A}, \mathbf{B} \in \mathbb{R}^{3 \times m}, \overline{\mathbf{R}} \in \mathrm{SO}(3)$ and for every $\chi, \psi \in W_{0}^{1, \infty}\left(D, \mathbb{R}^{3}\right)$.

Proof. We have to prove that

$$
f\left(\mathbf{A}, \overline{\mathbf{R}}_{0}, \mathbf{B}\right) \leq \frac{1}{\operatorname{meas}(D)} \int_{D} f\left(\mathbf{A}+\nabla \chi(x), \overline{\mathbf{R}}_{0}, \mathbf{B}+\nabla \boldsymbol{\psi}(x)\right) \mathrm{d} x
$$

for all $\mathbf{A}, \mathbf{B} \in \mathbb{R}^{3 \times m}, \overline{\mathbf{R}}_{0} \in \mathrm{SO}(3)$ and $\boldsymbol{\chi}, \boldsymbol{\psi} \in W_{0}^{1, \infty}\left(D ; \mathbb{R}^{3}\right)$ where $D$ is some cube. It is enough to establish (3.3) for $\boldsymbol{\chi}, \boldsymbol{\psi} \in C_{0}^{\infty}\left(D ; \mathbb{R}^{3}\right)$ by Remark 3.5.

We look at the case $m=3$ (the proof is the same for $m=1,2$ ). Let us denote the vector columns of the matrix $\mathbf{B}$ by $\boldsymbol{b}_{1}, \boldsymbol{b}_{2}, \boldsymbol{b}_{3}$, i.e. $\mathbf{B}=\left[\begin{array}{lll}\boldsymbol{b}_{1} & \boldsymbol{b}_{2} & \boldsymbol{b}_{3}\end{array}\right]$. Let $D=[0, \alpha]^{3} \subset \Omega$ and let $\boldsymbol{\chi}, \boldsymbol{\psi} \in C_{0}^{\infty}\left(D, \mathbb{R}^{3}\right)$. We extend $\boldsymbol{\chi}, \boldsymbol{\psi}$ to $\mathbb{R}^{3}$ by periodicity. For $h \in \mathbb{N}$ denote $Q_{h} \equiv \frac{1}{h} D \equiv\left[0, \frac{\alpha}{h}\right]^{3}$. We define

$$
\boldsymbol{\varphi}(x)=\mathbf{A} x, \quad \overline{\mathbf{R}}(x)=\exp \left(\mathbf{A}_{x_{1} \boldsymbol{b}_{1}}\right) \exp \left(\mathbf{A}_{x_{2} \boldsymbol{b}_{2}}\right) \exp \left(\mathbf{A}_{x_{3} \boldsymbol{b}_{3}}\right) \overline{\mathbf{R}}_{0},
$$

where $x=\left(x_{1}, x_{2}, x_{3}\right)$. The functions $\boldsymbol{\varphi}, \overline{\mathbf{R}}$ are of class $C^{\infty}$ and $\boldsymbol{\omega}(0)=\mathbf{B}$. We also define

$$
\begin{aligned}
& \boldsymbol{\varphi}_{h}^{k}(x)= \begin{cases}\varphi(x) & x \in \Omega \backslash Q_{h} \\
\varphi(x)+\frac{1}{k h} \chi(k h x) & \text { otherwise }\end{cases}
\end{aligned}
$$

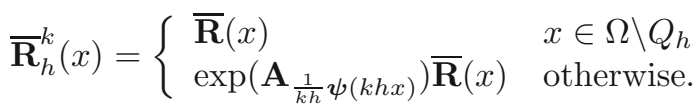

Using Lemma 3.7, for $h$ fixed, one has $\varphi_{h}^{k}=\boldsymbol{\varphi}, \overline{\mathbf{R}}_{h}^{k}=\overline{\mathbf{R}}$ on $\partial \Omega$ and

$$
\begin{aligned}
& \boldsymbol{\varphi}_{h}^{k} \rightarrow \boldsymbol{\varphi} \text { weak } * \text { in } W^{1, \infty}\left(\Omega, \mathbb{R}^{3}\right), \text { as } k \rightarrow \infty, \\
& \overline{\mathbf{R}}_{h}^{k} \rightarrow \overline{\mathbf{R}} \text { weak } * \text { in } W^{1, \infty}\left(\Omega, \mathbb{R}^{3 \times 3}\right), \text { as } k \rightarrow \infty .
\end{aligned}
$$


Since $\boldsymbol{\psi}, \boldsymbol{\chi} \in C_{0}^{\infty}\left(D, \mathbb{R}^{3}\right)$ and $\boldsymbol{\varphi}, \overline{\mathbf{R}}$ are $C^{\infty}$ it follows that $\overline{\mathbf{R}}_{h}^{k}, \boldsymbol{\varphi}_{h}^{k}$ are $C^{\infty}$. Moreover, their derivatives are uniformly bounded by a constant independent of $k$ (and $h$ ).

We now split $Q_{h}$ into cubes $Q_{h, j}^{k}, j=0, \ldots, k^{3}-1$ of length $\frac{\alpha}{k h}$ and denote by $P_{h, j}^{k}, j=0, \ldots, k^{3}-1$, the corner of $Q_{h, j}^{k}$ closest to 0 . Therefore

$$
Q_{h}=\bigcup_{j=0}^{k^{3}-1} Q_{h, j}^{k}=\bigcup_{j=0}^{k^{3}-1}\left(P_{h, j}^{k}+\frac{1}{k h} D\right)
$$

We now consider

$$
\begin{aligned}
I\left(\boldsymbol{\varphi}_{h}^{k}, \overline{\mathbf{R}}_{h}^{k}\right)= & \int_{\Omega} f\left(\nabla \boldsymbol{\varphi}_{h}^{k}(x), \overline{\mathbf{R}}_{h}^{k}(x), \boldsymbol{\omega}_{h}^{k}(x)\right) \mathrm{d} x \\
= & \int_{\Omega \backslash Q_{h}} f(\nabla \boldsymbol{\varphi}(x), \overline{\mathbf{R}}(x), \boldsymbol{\omega}(x)) \mathrm{d} x+\int_{Q_{h}} f\left(\nabla \boldsymbol{\varphi}_{h}^{k}(x), \overline{\mathbf{R}}_{h}^{k}(x), \boldsymbol{\omega}_{h}^{k}(x)\right) \mathrm{d} x \\
= & \int_{\Omega \backslash Q_{h}} f(\nabla \boldsymbol{\varphi}(x), \overline{\mathbf{R}}(x), \boldsymbol{\omega}(x)) \mathrm{d} x+\sum_{j=0}^{k^{3}-1} \int_{Q_{h, j}^{k}} f\left(\mathbf{A}+\nabla \chi(k h x), \overline{\mathbf{R}}_{h}^{k}(x), \boldsymbol{\omega}_{h}^{k}(x)\right) \mathrm{d} x \\
= & \int_{\Omega \backslash Q_{h}} f(\nabla \boldsymbol{\varphi}(x), \overline{\mathbf{R}}(x), \boldsymbol{\omega}(x)) \mathrm{d} x \\
& +\sum_{j=0}^{k^{3}-1} \int_{Q_{h, j}^{k}}\left[f\left(\mathbf{A}+\nabla \boldsymbol{\chi}(k h x), \overline{\mathbf{R}}_{h}^{k}(x), \boldsymbol{\omega}_{h}^{k}(x)\right)-f\left(\mathbf{A}+\nabla \chi(k h x), \overline{\mathbf{R}}_{h}^{k}(x), \boldsymbol{\omega}(x)+\nabla \boldsymbol{\psi}(k h x)\right)\right] \mathrm{d} x \\
& +\sum_{j=0}^{k^{3}-1} \int_{Q_{h, j}^{k}}\left[f\left(\nabla \boldsymbol{\varphi}_{h}^{k}(x), \overline{\mathbf{R}}_{h}^{k}(x), \boldsymbol{\omega}(x)+\nabla \boldsymbol{\psi}(k h x)\right)-f\left(\mathbf{A}+\nabla \boldsymbol{\chi}(k h x), \overline{\mathbf{R}}\left(P_{h, j}^{k}\right), \boldsymbol{\omega}\left(P_{h, j}^{k}\right)\right.\right. \\
& +\nabla \boldsymbol{\psi}(k h x)) \mathrm{d} x \\
& +\sum_{j=0}^{k^{3}-1} \int_{Q_{h, j}^{k}} f\left(\mathbf{A}+\nabla \boldsymbol{\chi}(k h x), \overline{\mathbf{R}}\left(P_{h, j}^{k}\right), \boldsymbol{\omega}\left(P_{h, j}^{k}\right)+\nabla \boldsymbol{\psi}(k h x)\right) \mathrm{d} x \\
= & I_{1}(k)+I_{2}(k)+I_{3}(k)+I_{4}(k) .
\end{aligned}
$$

In the sequel we consider the asymptotic of these terms, for fixed $h$, when $k \rightarrow \infty$.

Boundedness of $\boldsymbol{\omega}$ and (3.1) imply $\left\|\boldsymbol{\omega}_{h}^{k}(x)-\boldsymbol{\omega}(x)-\nabla \boldsymbol{\psi}(k h x)\right\| \rightarrow 0$, uniformly with respect to $x$. The uniform continuity of $f$ on a compact set and $L^{\infty}$ bounds on $\mathbf{A}+\nabla \boldsymbol{\chi}(k h x), \overline{\mathbf{R}}_{h}^{k}(x), \boldsymbol{\omega}_{h}^{k}(x), \boldsymbol{\omega}(x)+\nabla \boldsymbol{\psi}(k h x)$ imply $I_{2}(k) \rightarrow 0$.

The convergence $\overline{\mathbf{R}}_{h}^{k} \rightarrow \overline{\mathbf{R}}$ (by (3.2)), uniform continuity of $\overline{\mathbf{R}}, \boldsymbol{\omega}$, uniform continuity of $f$ on a compact set in the same way imply $I_{3}(k) \rightarrow 0$.

As $Q_{h, j}^{k}=P_{h, j}^{k}+\frac{1}{k h} D$, using periodicity of $\boldsymbol{\psi}, \boldsymbol{\chi}$, one has

$$
I_{4}(k)=\sum_{j=0}^{k^{3}-1} \frac{1}{(k h)^{3}} \int_{D} f\left(\mathbf{A}+\nabla \boldsymbol{\chi}(y), \overline{\mathbf{R}}\left(P_{h, j}^{k}\right), \boldsymbol{\omega}\left(P_{h, j}^{k}\right)+\nabla \boldsymbol{\psi}(y)\right) \mathrm{d} y .
$$

Recognizing the Riemann sum of the continuous function in the last expression, we obtain

$$
\lim _{k \rightarrow \infty} I_{4}(k)=\frac{1}{\operatorname{meas}(D)} \int_{Q_{h}} \int_{D} f(\mathbf{A}+\nabla \boldsymbol{\chi}(y), \overline{\mathbf{R}}(x), \boldsymbol{\omega}(x)+\nabla \boldsymbol{\psi}(y)) \mathrm{d} y \mathrm{~d} x .
$$


Therefore by assumption of the theorem we obtain

$$
\begin{aligned}
\liminf _{k \rightarrow \infty} I\left(\varphi_{h}^{k}, \overline{\mathbf{R}}_{h}^{k}\right)= & \int_{\Omega \backslash Q_{h}} f(\nabla \varphi(x), \overline{\mathbf{R}}(x), \boldsymbol{\omega}(x)) \mathrm{d} x \\
& +\frac{1}{\operatorname{meas}(D)} \int_{Q_{h}} \int_{D} f(\mathbf{A}+\nabla \chi(y), \overline{\mathbf{R}}(x), \boldsymbol{\omega}(x)+\nabla \boldsymbol{\psi}(y)) \mathrm{d} y \mathrm{~d} x \\
\geq & I(\boldsymbol{\varphi}, \overline{\mathbf{R}})=\int_{\Omega} f(\nabla \boldsymbol{\varphi}(x), \overline{\mathbf{R}}(x), \boldsymbol{\omega}(x)) \mathrm{d} x
\end{aligned}
$$

and hence

$$
\frac{1}{\operatorname{meas}\left(Q_{h}\right)} \int_{Q_{h}} \int_{D} f(\mathbf{A}+\nabla \boldsymbol{\chi}(y), \overline{\mathbf{R}}(x), \boldsymbol{\omega}(x)+\nabla \boldsymbol{\psi}(y)) \mathrm{d} y \mathrm{~d} x \geq \frac{\operatorname{meas}(D)}{\operatorname{meas}\left(Q_{h}\right)} \int_{Q_{h}} f(\mathbf{A}, \overline{\mathbf{R}}(x), \boldsymbol{\omega}(x)) \mathrm{d} x .
$$

Now letting $h \rightarrow \infty$ and using continuity and the fact that $\overline{\mathbf{R}}(0)=\overline{\mathbf{R}}_{0}, \boldsymbol{\omega}(0)=\mathbf{B}$ we have the claim.

\section{SufFICIENCY OF QUASICONVEXITY}

In the sequel we prove the sufficiency of quasiconvexity for sequentially weakly lower semicontinuity. We shall impose some conditions on the function $f$, but as we shall see these conditions are consequence of objectivity of the function $f$. The main drawback is that we impose condition $p>m$.

The following lemma is crucial for the proof of the sufficiency of the quasiconvexity. It tells us that for every weakly convergent sequence of rotations $\overline{\mathbf{R}}^{k}$ to the rotation $\overline{\mathbf{R}}$ in the space $W^{1, p}(\Omega, \mathrm{SO}(3)), p>m$ the variables $\boldsymbol{\omega}^{k}$ are essentially of the form $\boldsymbol{\omega}+\nabla \mathbf{v}^{k}$, where $\mathbf{v}^{k} \rightarrow 0$ in $W^{1, p}\left(\Omega, \mathbb{R}^{3}\right)$. This establishes the analogy between $\boldsymbol{\omega}$ and $\nabla \varphi$, since $\nabla \varphi^{k}=\nabla \varphi+\nabla\left(\varphi^{k}-\varphi\right)$. Some kind of analogy was already established in Lemma 3.7, although the specific oscillations of rotations are chosen there.

Lemma 4.1. Let $\Omega$ be an open bounded set with the Lipschitz boundary, $\Omega \subset \mathbb{R}^{m}$. Let $\overline{\mathbf{R}}^{k} \in W^{1, p}(\Omega, \mathrm{SO}(3))$ converges weakly to $\overline{\mathbf{R}}$ in $W^{1, p}\left(\Omega, \mathbb{R}^{3 \times 3}\right)$, where $m<p \leq \infty$. Then there exist a sequence $\left(\mathbf{s}^{k}\right)_{k}$ which converges to 0 strongly in $L^{p}\left(\Omega, \mathbb{R}^{3 \times m}\right)$ and a sequence $\left(\boldsymbol{v}^{k}\right)_{k}$ which converges weakly (weak $*$ if $\left.p=\infty\right)$ to 0 in $W^{1, p}\left(\Omega, \mathbb{R}^{3}\right.$ ) such that $\boldsymbol{\omega}^{k}=\boldsymbol{\omega}+\nabla \boldsymbol{v}^{k}+\mathbf{s}^{k}$, where

$$
\boldsymbol{\omega}_{i}^{k}=\frac{1}{2} \overline{\boldsymbol{R}}_{j}^{k} \times \partial_{i} \overline{\boldsymbol{R}}_{j}^{k}, \boldsymbol{\omega}_{i}=\frac{1}{2} \overline{\boldsymbol{R}}_{j} \times \partial_{i} \overline{\boldsymbol{R}}_{j} .
$$

Proof. By direct calculation we obtain

$$
\boldsymbol{\omega}_{i}^{k}=\boldsymbol{\omega}_{i}+\partial_{i}\left(\frac{1}{2} \overline{\boldsymbol{R}}_{j} \times \overline{\boldsymbol{R}}_{j}^{k}\right)+\left(\overline{\boldsymbol{R}}_{j}^{k}-\overline{\boldsymbol{R}}_{j}\right) \times \partial_{i} \overline{\boldsymbol{R}}_{j}+\frac{1}{2}\left(\overline{\boldsymbol{R}}_{j}^{k}-\overline{\boldsymbol{R}}_{j}\right) \times \partial_{i}\left(\overline{\boldsymbol{R}}_{j}^{k}-\overline{\boldsymbol{R}}_{j}\right) .
$$

Let

$$
\boldsymbol{v}^{k}=\frac{1}{2} \overline{\boldsymbol{R}}_{j} \times \overline{\boldsymbol{R}}_{j}^{k}, \mathbf{s}_{i}^{k}=\left(\overline{\boldsymbol{R}}_{j}^{k}-\overline{\boldsymbol{R}}_{j}\right) \times \partial_{i} \overline{\boldsymbol{R}}_{j}+\frac{1}{2}\left(\overline{\boldsymbol{R}}_{j}^{k}-\overline{\boldsymbol{R}}_{j}\right) \times \partial_{i}\left(\overline{\boldsymbol{R}}_{j}^{k}-\overline{\boldsymbol{R}}_{j}\right) .
$$

By the Sobolev embedding theorem $\left\|\overline{\boldsymbol{R}}_{j}^{k}-\overline{\boldsymbol{R}}_{j}\right\|_{L^{\infty}\left(\Omega ; \mathbb{R}^{3 \times 3}\right)}$ converges to 0 . Using it and the boundedness of $\left(\overline{\mathbf{R}}^{k}\right)_{k} \subset W^{1, p}\left(\Omega ; \mathbb{R}^{3 \times 3}\right)$ the statement of the lemma follows.

The following lemma is known in the measure theory.

Lemma 4.2. Let $\Omega$ be a finite union of open cubes and $f \in L^{p}(\Omega ; \mathbb{R}), p \in[1, \infty\rangle$. For each $n \in \mathbb{N}$ we divide $\Omega$ in a finite union of cubes $D_{s}$ of diameter less or equal $1 / n$ and define numbers

$$
A_{s}=\frac{1}{\operatorname{meas}\left(D_{s}\right)} \int_{D_{s}} f(x) \mathrm{d} x .
$$


Then the sequence of functions defined by

$$
f^{n}(x)=\sum_{s} A_{s} 1_{D_{s}}(x)
$$

converges strongly to $f$ in $L^{p}(\Omega ; \mathbb{R})$.

Remark 4.3. For $p=\infty$ we can just conclude that $\left\|f^{n}\right\|_{L^{\infty}(\Omega)} \leq\|f\|_{L^{\infty}(\Omega)}$ and that $f^{n}(x) \rightarrow f(x)$ for a.e. $x \in \Omega$ (see [8], p. 93).

We now turn ourselves to the main theorem. As a first step we consider the case when $f$ depends only on $\nabla \boldsymbol{\varphi}, \boldsymbol{\omega}$ (which is physically unrealistic since the fields of deformation and microrotation completely decouple). We adapt the proof from [6], Theorem 2.3, p. 158, using Lemma 4.1.

Theorem 4.4. Let $\Omega \subset \mathbb{R}^{m}$ be an open bounded set with the Lipschitz boundary and $m<p<\infty$. Let $f: \mathbb{R}^{3 \times m} \times \mathbb{R}^{3 \times m} \rightarrow \mathbb{R}$ be a quasiconvex function and that there are $K>0$ and $\beta \geq 0$ such that

(a) $f(\mathbf{A}, \mathbf{B})<K\left(1+\|\mathbf{A}\|^{p}+\|\mathbf{B}\|^{p}\right)$,

(b) $\left|f\left(\mathbf{A}_{1}, \mathbf{B}_{1}\right)-f\left(\mathbf{A}_{2}, \mathbf{B}_{2}\right)\right|<K\left(1+\left\|\mathbf{A}_{1}\right\|^{p-1}+\left\|\mathbf{B}_{1}\right\|^{p-1}+\left\|\mathbf{A}_{2}\right\|^{p-1}+\left\|\mathbf{B}_{2}\right\|^{p-1}\right)\left(\left\|\mathbf{A}_{1}-\mathbf{A}_{2}\right\|+\left\|\mathbf{B}_{1}-\mathbf{B}_{2}\right\|\right)$,

(c) $f(\mathbf{A}, \mathbf{B}) \geq-\beta$.

Let

$$
I(\boldsymbol{\varphi}, \overline{\mathbf{R}})=\int_{\Omega} f(\nabla \varphi(x), \boldsymbol{\omega}(x)) \mathrm{d} x .
$$

Then for every sequence $\left(\left(\varphi^{k}, \overline{\mathbf{R}}^{k}\right)\right)_{k} \subset W^{1, p}\left(\Omega, \mathbb{R}^{3}\right) \times W^{1, p}(\Omega, \mathrm{SO}(3))$ which converges weakly to $(\boldsymbol{\varphi}, \overline{\mathbf{R}})$ in $W^{1, p}\left(\Omega, \mathbb{R}^{3}\right) \times W^{1, p}\left(\Omega, \mathbb{R}^{3 \times 3}\right)$ one has

$$
I(\varphi, \overline{\mathbf{R}}) \leq \liminf _{k \rightarrow \infty} I\left(\varphi^{k}, \overline{\mathbf{R}}^{k}\right)
$$

Proof. Let us take $\left(\varphi^{k}, \overline{\mathbf{R}}^{k}\right) \in W^{1, p}\left(\Omega, \mathbb{R}^{3}\right) \times W^{1, p}(\Omega, \mathrm{SO}(3))$ such that

$$
\left(\varphi^{k}, \overline{\mathbf{R}}^{k}\right) \rightarrow(\varphi, \overline{\mathbf{R}}) \text { weakly in } W^{1, p}\left(\Omega, \mathbb{R}^{3}\right) \times W^{1, p}\left(\Omega ; \mathbb{R}^{3 \times 3}\right) .
$$

Using Lemma 4.1 we form the sequences $\left(\mathbf{s}^{k}\right)_{k},\left(\boldsymbol{v}^{k}\right)_{k}$ such that $\mathbf{s}^{k}$ converges to 0 strongly in $L^{p}\left(\Omega ; \mathbb{R}^{3 \times m}\right), \boldsymbol{v}^{k}$ which converges weakly to 0 in $W^{1, p}\left(\Omega ; \mathbb{R}^{3}\right)$ and $\boldsymbol{\omega}^{k}=\boldsymbol{\omega}+\nabla \boldsymbol{v}^{k}+\mathbf{s}^{k}$.

Let $\varepsilon>0$. Then there exist $\delta$ such that

$$
\operatorname{meas}(S)<\delta \Rightarrow \int_{S}|f(\nabla \varphi, \omega)|<\varepsilon
$$

This is possible because, due to the conditions (a) and (c), the mapping $x \rightarrow f(\nabla \varphi(x), \boldsymbol{\omega}(x))$ is in $L^{1}(\Omega)$. We then approximate $\Omega$ by a union of cubes $D_{s}$ whose edge length is $\frac{1}{2^{N}}$; we denote this union by $H_{N}=\cup_{s} D_{s}$. We then choose $N$ large enough so that

$$
\operatorname{meas}\left(\Omega \backslash H_{N}\right) \leq \min \left(\frac{\varepsilon}{\beta}, \delta\right)
$$

Let

$$
\mathbf{A}_{s}=\frac{1}{\operatorname{meas}\left(D_{s}\right)} \int_{D_{s}} \nabla \boldsymbol{\varphi}(x) \mathrm{d} x, \quad \mathbf{B}_{s}=\frac{1}{\operatorname{meas}\left(D_{s}\right)} \int_{D_{s}} \boldsymbol{\omega}(x) \mathrm{d} x .
$$

Since $(\nabla \boldsymbol{\varphi}, \boldsymbol{\omega}) \in L^{p}\left(\Omega ; \mathbb{R}^{3 \times m} \times \mathbb{R}^{3 \times m}\right)$ we can choose $N$ larger if necessarily such that

$$
\sum_{s} \int_{D_{s}}\left\|\nabla \boldsymbol{\varphi}(x)-\mathbf{A}_{s}\right\|^{p}+\sum_{s} \int_{D_{s}}\left\|\boldsymbol{\omega}-\mathbf{B}_{s}\right\|^{p}<\frac{\varepsilon^{p}}{M^{p}}
$$


by Lemma 4.2 . Here the constant $M$ is chosen to satisfy

$$
M^{\frac{p}{p-1}}>\int_{\Omega}\left(1+\left\|\nabla \boldsymbol{\varphi}^{k}\right\|^{p}+\left\|\boldsymbol{\omega}+\nabla \boldsymbol{v}^{k}\right\|^{p}+\left\|\mathbf{A}_{s}\right\|^{p}+\left\|\nabla \boldsymbol{\varphi}^{k}-\nabla \boldsymbol{\varphi}\right\|^{p}+\left\|\mathbf{B}_{s}\right\|^{p}+\left\|\nabla \boldsymbol{v}^{k}\right\|^{p}\right) \mathrm{d} x
$$

for every $k$. Such $M$ exists as all the terms on the right hand side are bounded either as they are weakly convergent or by $\int_{H_{N}}\left\|\mathbf{A}_{s}\right\|^{p} \leq \int_{H_{N}}\|\nabla \boldsymbol{\varphi}\|^{p}$ and $\int_{H_{N}}\left\|\mathbf{B}_{s}\right\|^{p} \leq \int_{H_{N}}\|\boldsymbol{\omega}\|^{p}$.

We now consider

$$
\begin{aligned}
I\left(\boldsymbol{\varphi}^{k}, \overline{\mathbf{R}}^{k}\right)-I(\boldsymbol{\varphi}, \overline{\mathbf{R}})= & \int_{\Omega}\left[f\left(\nabla \boldsymbol{\varphi}^{k}(x), \boldsymbol{\omega}^{k}(x)\right)-f(\nabla \boldsymbol{\varphi}(x), \boldsymbol{\omega}(x))\right] \mathrm{d} x \\
= & \int_{\Omega}\left[f\left(\nabla \boldsymbol{\varphi}^{k}(x), \boldsymbol{\omega}(x)+\nabla \mathbf{v}^{k}(x)+\mathbf{s}^{k}(x)\right)-f\left(\nabla \boldsymbol{\varphi}^{k}(x), \boldsymbol{\omega}(x)+\nabla \mathbf{v}^{k}(x)\right)\right] \mathrm{d} x \\
& \left.+\int_{\Omega} f\left(\nabla \boldsymbol{\varphi}^{k}(x), \boldsymbol{\omega}(x)+\nabla \mathbf{v}^{k}(x)\right)-f(\nabla \boldsymbol{\varphi}(x), \boldsymbol{\omega}(x))\right] \mathrm{d} x \\
= & J_{1}(k)+J_{2}(k) .
\end{aligned}
$$

Using the condition (b) and the Hölder inequality we estimate the first integral

$$
\begin{aligned}
J_{1}(k) & \leq \int_{\Omega} K\left(1+2\left\|\nabla \boldsymbol{\varphi}^{k}\right\|^{p-1}+\left\|\boldsymbol{\omega}+\nabla \mathbf{v}^{k}+\mathbf{s}^{k}\right\|^{p-1}+\left\|\boldsymbol{\omega}+\boldsymbol{v}^{k}\right\|^{p-1}\right)\left\|\mathbf{s}^{k}\right\| \\
& \leq K_{1}\left(\int_{\Omega} 1+2\left\|\nabla \boldsymbol{\varphi}^{k}\right\|^{p}+\left\|\boldsymbol{\omega}+\nabla \boldsymbol{v}^{k}+\mathbf{s}^{k}\right\|^{p}+\left\|\boldsymbol{\omega}+\boldsymbol{v}^{k}\right\|^{p}\right)^{\frac{p-1}{p}}\left(\int_{\Omega}\left\|\mathbf{s}^{k}\right\|^{p}\right)^{\frac{1}{p}} .
\end{aligned}
$$

As it weakly converges the first factor is bounded. Moreover the second factor converges to 0 . Thus $J_{1}(k)$ converges to 0 , so for $k$ large enough $\left|J_{1}(k)\right|<\varepsilon$. We now proceed to estimate $J_{2}(k)$ :

$$
\begin{aligned}
J_{2}(k)= & \int_{\Omega \backslash H_{N}}\left[f\left(\nabla \boldsymbol{\varphi}^{k}(x), \boldsymbol{\omega}(x)+\nabla \boldsymbol{v}^{k}(x)\right)-f(\nabla \boldsymbol{\varphi}(x), \boldsymbol{\omega}(x))\right] \mathrm{d} x \\
& +\sum_{s} \int_{D_{s}}\left[f\left(\nabla \boldsymbol{\varphi}(x)+\left(\nabla \boldsymbol{\varphi}^{k}(x)-\nabla \boldsymbol{\varphi}(x)\right), \boldsymbol{\omega}(x)+\nabla \boldsymbol{v}^{k}(x)\right)\right. \\
& \left.-f\left(\mathbf{A}_{s}+\left(\nabla \boldsymbol{\varphi}^{k}(x)-\nabla \boldsymbol{\varphi}(x)\right), \mathbf{B}_{s}+\nabla \boldsymbol{v}^{k}(x)\right)\right] \mathrm{d} x \\
& +\sum_{s} \int_{D_{s}}\left[f\left(\mathbf{A}_{s}+\left(\nabla \boldsymbol{\varphi}^{k}(x)-\nabla \boldsymbol{\varphi}(x)\right), \mathbf{B}_{s}+\nabla \boldsymbol{v}^{k}(x)\right)-f\left(\mathbf{A}_{s}, \mathbf{B}_{s}\right)\right] \mathrm{d} x \\
& +\sum_{s} \int_{D_{s}}\left[f\left(\mathbf{A}_{s}, \mathbf{B}_{s}\right)-f(\nabla \boldsymbol{\varphi}(x), \boldsymbol{\omega}(x))\right] \mathrm{d} x \\
= & J_{3}(k)+J_{4}(k)+J_{5}(k)+J_{6}(k) .
\end{aligned}
$$

Since $f \geq-\beta$, using the definition of $\delta$ and $N$, we have

$$
J_{3}(k) \geq-\beta \operatorname{meas}\left(\Omega \backslash H_{N}\right)-\int_{\Omega \backslash H_{N}} f(\nabla \varphi(x), \boldsymbol{\omega}(x)) \mathrm{d} x>-2 \varepsilon .
$$


Using the condition (b) we obtain

$$
\begin{aligned}
J_{4}(k) \leq & \sum_{s} \int_{D_{s}} K\left(1+\left\|\nabla \boldsymbol{\varphi}^{k}\right\|^{p-1}+\left\|\boldsymbol{\omega}+\nabla \boldsymbol{v}^{k}\right\|^{p-1}+\left\|\mathbf{A}_{s}+\nabla \boldsymbol{\varphi}^{k}-\nabla \boldsymbol{\varphi}\right\|^{p-1}\right. \\
& \left.+\left\|\mathbf{B}_{s}+\nabla \boldsymbol{v}^{k}\right\|^{p-1}\right)\left(\left\|\nabla \boldsymbol{\varphi}-\mathbf{A}_{s}\right\|+\left\|\boldsymbol{\omega}-\mathbf{B}_{s}\right\|\right) \\
\leq & K_{1}(p) \sum_{s}\left(\int_{D_{s}} 1+\left\|\nabla \boldsymbol{\varphi}^{k}\right\|^{p}+\left\|\boldsymbol{\omega}+\nabla \boldsymbol{v}^{k}\right\|^{p}+\left\|\mathbf{A}_{s}\right\|^{p}+\left\|\nabla \boldsymbol{\varphi}^{k}-\nabla \boldsymbol{\varphi}\right\|^{p}\right. \\
& \left.+\left\|\mathbf{B}_{s}\right\|^{p}+\left\|\nabla \boldsymbol{v}^{k}\right\|^{p}\right)^{\frac{p-1}{p}}\left(\int_{D_{s}}\left\|\nabla \boldsymbol{\varphi}-\mathbf{A}_{s}\right\|^{p}+\left\|\boldsymbol{\omega}-\mathbf{B}_{s}\right\|^{p}\right)^{\frac{1}{p}} \\
\leq & K_{1}(p)\left(\int _ { H _ { N } } \left(1+\left\|\nabla \boldsymbol{\varphi}^{k}\right\|^{p}+\left\|\boldsymbol{\omega}+\nabla \boldsymbol{v}^{k}\right\|^{p}+\left\|\mathbf{A}_{s}\right\|^{p}+\left\|\nabla \boldsymbol{\varphi}^{k}-\nabla \boldsymbol{\varphi}\right\|^{p}\right.\right. \\
& \left.\left.+\left\|\mathbf{B}_{s}\right\|^{p}+\left\|\nabla \boldsymbol{v}^{k}\right\|^{p}\right)\right)^{\frac{p-1}{p}}\left(\int_{H_{N}}\left\|\nabla \boldsymbol{\varphi}-\mathbf{A}_{s}\right\|^{p}+\left\|\boldsymbol{\omega}-\mathbf{B}_{s}\right\|^{p}\right)^{\frac{1}{p}} \\
< & K_{1}(p) M \frac{\varepsilon}{M}=K_{1}(p) \varepsilon,
\end{aligned}
$$

where we have used the Hölder inequality on each $D_{s}$ and the Hölder inequality for numbers.

In the same way we can estimate $J_{6}(k)$. Estimation of $J_{5}(k)$ is essential. We have to prove that for $\varphi^{k}=\varphi^{k}-\boldsymbol{\varphi}, \boldsymbol{v}^{k}$ which converge weakly to 0 in $W^{1, p}\left(\Omega ; \mathbb{R}^{3}\right)$ and for $D$ an arbitrary cube in $\mathbb{R}^{m}$ one has

$$
\liminf _{k \rightarrow \infty} \int_{D} f\left(\mathbf{A}+\nabla \varphi^{k}(x), \mathbf{B}+\nabla \boldsymbol{v}^{k}(x)\right) \mathrm{d} x \geq f(\mathbf{A}, \mathbf{B}) \operatorname{meas}(D)
$$

we have to infer it from the quasiconvexity (see [6], pp. 163-166).

Therefore for all $\varepsilon>0$

$$
\liminf _{k \rightarrow \infty} I\left(\varphi^{k}, \overline{\mathbf{R}}^{k}\right)-I(\boldsymbol{\varphi}, \overline{\mathbf{R}}) \geq-2 \varepsilon
$$

which implies the statement of the theorem.

Remark 4.5. In case $p=\infty$ the same holds without the assumptions (a), (b) and (c). The integrals $J_{1}(k)$, $J_{4}(k), J_{6}(k) \rightarrow 0$ by the Lebesgue theorem of the dominated convergence, the uniform continuity of $f$ on bounded subsets of $\mathbb{R}^{3 \times m} \times \mathbb{R}^{3 \times m}$ and Remark 4.3.

We now turn ourselves in the general functionals of the type

$$
I(\boldsymbol{\varphi}, \overline{\mathbf{R}})=\int_{\Omega} f(\nabla \boldsymbol{\varphi}(x), \overline{\mathbf{R}}(x), \boldsymbol{\omega}(x)) \mathrm{d} x .
$$

We impose the following assumptions on the function $f$ :

(a) $f(\mathbf{A}, \overline{\mathbf{R}}, \mathbf{B}) \leq K\left(1+\|\mathbf{A}\|^{p}+\|\mathbf{B}\|^{p}\right), \quad \mathbf{A}, \mathbf{B} \in \mathbb{R}^{3 \times m}, \overline{\mathbf{R}} \in \mathrm{SO}(3)$;

(b) $\left|f\left(\mathbf{A}_{1}, \overline{\mathbf{R}}, \mathbf{B}_{1}\right)-f\left(\mathbf{A}_{2}, \overline{\mathbf{R}}, \mathbf{B}_{2}\right)\right| \leq K\left(1+\left\|\mathbf{A}_{1}\right\|^{p-1}+\left\|\mathbf{B}_{1}\right\|^{p-1}+\left\|\mathbf{A}_{2}\right\|^{p-1}+\left\|\mathbf{B}_{2}\right\|^{p-1}\right)\left(\left\|\mathbf{A}_{1}-\mathbf{A}_{2}\right\|+\| \mathbf{B}_{1}-\right.$ $\left.\mathbf{B}_{2} \|\right), \quad \mathbf{A}_{1}, \mathbf{A}_{2}, \mathbf{B}_{1}, \mathbf{B}_{2} \in \mathbb{R}^{3 \times m}, \overline{\mathbf{R}} \in \mathrm{SO}(3) ;$

(c) $f \geq-\beta$, for some $\beta \geq 0$;

(d) $\left|f\left(\mathbf{A}, \overline{\mathbf{R}}_{1}, \mathbf{B}\right)-f\left(\mathbf{A}, \overline{\mathbf{R}}_{2}, \mathbf{B}\right)\right| \leq K\left(1+\|\mathbf{A}\|^{p}+\|\mathbf{B}\|^{p}\right)\left\|\overline{\mathbf{R}}_{1}-\overline{\mathbf{R}}_{2}\right\|, \quad \mathbf{A}, \mathbf{B} \in \mathbb{R}^{3 \times m}, \overline{\mathbf{R}}_{1}, \overline{\mathbf{R}}_{2} \in \operatorname{SO}(3)$.

We follow [6], pp. 167-169.

Theorem 4.6. Let $\Omega \subset \mathbb{R}^{m}$ be an open bounded set with the Lipschitz boundary and $m<p<\infty$. Let $f: \mathbb{R}^{3 \times m} \times \mathrm{SO}(3) \times \mathbb{R}^{3 \times m} \rightarrow \mathbb{R}$ be quasiconvex in the first and the last variable and satisfies (a)-(d). Let

$$
I(\boldsymbol{\varphi}, \overline{\mathbf{R}})=\int_{\Omega} f(\nabla \boldsymbol{\varphi}(x), \overline{\mathbf{R}}(x), \boldsymbol{\omega}(x)) \mathrm{d} x .
$$


Then for every sequence $\left(\left(\varphi^{k}, \overline{\mathbf{R}}^{k}\right)\right)_{k} \subset W^{1, p}\left(\Omega, \mathbb{R}^{3}\right) \times W^{1, p}(\Omega, \operatorname{SO}(3))$ which converges weakly to $(\boldsymbol{\varphi}, \overline{\mathbf{R}})$ in $W^{1, p}\left(\Omega, \mathbb{R}^{3}\right) \times W^{1, p}\left(\Omega, \mathbb{R}^{3 \times 3}\right)$ one has

$$
I(\varphi, \overline{\mathbf{R}}) \leq \liminf _{k} I\left(\varphi^{k}, \overline{\mathbf{R}}^{k}\right)
$$

Proof. Let $\varepsilon>0$ and define $H_{N}, D_{s}$ as in the proof of Theorem 4.4 (note that $x \rightarrow f(\nabla \varphi(x), \overline{\mathbf{R}}(x), \boldsymbol{\omega}(x)$ ) is an integrable function). Since, by the Sobolev embedding theorem, $\overline{\mathbf{R}}$ is continuous on $\bar{\Omega}$ in construction of $D_{s}$ we can choose $N$ large enough such that

$$
\left\|\overline{\mathbf{R}}(x)-\overline{\mathbf{R}}\left(x_{s}\right)\right\| \leq \frac{\varepsilon}{K M}, \quad x \in D_{s},
$$

for every $s$, where $x_{s}$ is the center of $D_{s}$. Here $K$ is the constant from the conditions (a), (b) and (d) and $M$ is such that

$$
1+2 \int_{\Omega}\left\|\nabla \varphi^{k}(x)\right\|^{p}+2 \int_{\Omega}\|\nabla \varphi(x)\|^{p}+2 \int_{\Omega}\left\|\omega^{k}(x)\right\|^{p}+2 \int_{\Omega}\|\boldsymbol{\omega}(x)\|^{p}<M
$$

such $M$ exists due to the weak convergence in $L^{p}$ of the functions on the left hand side. Then one has

$$
\begin{aligned}
I\left(\boldsymbol{\varphi}^{k}, \overline{\mathbf{R}}^{k}\right)-I(\boldsymbol{\varphi}, \overline{\mathbf{R}})= & \int_{\Omega \backslash H_{N}}\left[f\left(\nabla \boldsymbol{\varphi}^{k}(x), \overline{\mathbf{R}}^{k}(x), \boldsymbol{\omega}^{k}(x)\right)-f(\nabla \boldsymbol{\varphi}(x), \overline{\mathbf{R}}(x), \boldsymbol{\omega}(x))\right] \mathrm{d} x \\
& +\int_{H_{N}}\left[f\left(\nabla \boldsymbol{\varphi}^{k}(x), \overline{\mathbf{R}}^{k}(x), \boldsymbol{\omega}^{k}(x)\right)-f\left(\nabla \boldsymbol{\varphi}^{k}(x), \overline{\mathbf{R}}(x), \boldsymbol{\omega}^{k}(x)\right)\right] \mathrm{d} x \\
& +\sum_{s} \int_{D_{s}}\left[f\left(\nabla \boldsymbol{\varphi}^{k}(x), \overline{\mathbf{R}}(x), \boldsymbol{\omega}^{k}(x)\right)-f\left(\nabla \boldsymbol{\varphi}^{k}(x), \overline{\mathbf{R}}\left(x_{s}\right), \boldsymbol{\omega}^{k}(x)\right)\right] \mathrm{d} x \\
& +\sum_{s} \int_{D_{s}}\left[f\left(\nabla \boldsymbol{\varphi}^{k}(x), \overline{\mathbf{R}}\left(x_{s}\right), \boldsymbol{\omega}^{k}(x)\right)-f\left(\nabla \boldsymbol{\varphi}(x), \overline{\mathbf{R}}\left(x_{s}\right), \boldsymbol{\omega}(x)\right)\right] \mathrm{d} x \\
& +\sum_{s} \int_{D_{s}}\left[f\left(\nabla \boldsymbol{\varphi}(x), \overline{\mathbf{R}}\left(x_{s}\right), \boldsymbol{\omega}(x)\right)-f(\nabla \boldsymbol{\varphi}(x), \overline{\mathbf{R}}(x), \boldsymbol{\omega}(x))\right] \mathrm{d} x \\
= & J_{1}(k)+J_{2}(k)+J_{3}(k)+J_{4}(k)+J_{5}(k) .
\end{aligned}
$$

Using the same arguments as in the proof of Theorem 4.4 (for $J_{3}(k)$ ) we accomplish that $J_{1}(k)>-2 \varepsilon$ for all $k$. Let us estimate $J_{2}(k)$. Using the condition (d) we have that

$$
\left|J_{2}(k)\right|<K \int_{H_{N}}\left(1+\left\|\nabla \boldsymbol{\varphi}^{k}\right\|^{p}+\|\nabla \boldsymbol{\varphi}\|^{p}+\left\|\boldsymbol{\omega}^{k}\right\|^{p}+\|\boldsymbol{\omega}\|^{p}\right)\left\|\overline{\mathbf{R}}^{k}-\overline{\mathbf{R}}\right\| .
$$

Since $\overline{\mathbf{R}}^{k} \rightarrow \overline{\mathbf{R}}$ uniformly on $\bar{\Omega}$, by the Sobolev embedding theorem, we can choose $k$ large enough such that $\left|J_{2}(k)\right|<\varepsilon$. Again using (d) we obtain that for $k$ large enough $\left|J_{3}(k)\right|,\left|J_{5}(k)\right|<\varepsilon$.

Estimation of $J_{4}(k)$ is essential. Using Theorem 4.4 on each $D_{s}$ we obtain

$$
\liminf _{k \rightarrow \infty} \int_{D_{s}} f\left(\nabla \varphi^{k}(x), \overline{\mathbf{R}}\left(x_{s}\right), \boldsymbol{\omega}^{k}(x)\right) \mathrm{d} x-\int_{D_{s}} f\left(\nabla \varphi(x), \overline{\mathbf{R}}\left(x_{s}\right), \boldsymbol{\omega}(x)\right) \mathrm{d} x \geq 0 .
$$

Therefore $\liminf _{k \rightarrow \infty} J_{4}(k) \geq 0$, so for all $\varepsilon>0$ we obtain

$$
\liminf _{k \rightarrow \infty} I\left(\varphi^{k}, \overline{\mathbf{R}}^{k}\right)-I(\varphi, \overline{\mathbf{R}}) \geq-5 \varepsilon
$$

which implies the statement of the theorem. 
Remark 4.7. In case $p=\infty$ we only need the continuity of $f$ and quasiconvexity in the first and the last variable of $f$.

Now we shall see that the properties (b) and (d) are consequences of objectivity and quasiconvexity. This enables us to restate Theorem 4.6 with assumptions (b) and (d) replaced by objectivity of $f$. First we state one helpful lemma (see [6], p. 156).

Lemma 4.8. Let $f: \mathbb{R}^{n} \rightarrow \mathbb{R}$ be convex in each variable and $\alpha \geq 0, p \geq 1$. Let

$$
|f(x)| \leq \alpha\left(1+\|x\|^{p}\right), \quad x \in \mathbb{R}^{n} .
$$

Then there exists $\gamma \geq 0$ such that:

$$
|f(x)-f(y)| \leq \gamma\left(1+\|x\|^{p-1}+\|y\|^{p-1}\right)\|x-y\|, \quad x, y \in \mathbb{R}^{n} .
$$

Proposition 4.9. Let $f: \mathbb{R}^{3 \times m} \times \mathrm{SO}(3) \times \mathbb{R}^{3 \times m} \rightarrow \mathbb{R}$ be objective quasiconvex stored energy function which satisfies growth condition

Then there exists $g: \mathbb{R}^{3 \times m} \times \mathbb{R}^{3 \times m} \rightarrow \mathbb{R}$ such that

$$
|f(\mathbf{A}, \overline{\mathbf{R}}, \mathbf{B})| \leq K\left(1+\|\mathbf{A}\|^{p}+\|\mathbf{B}\|^{p}\right) .
$$

(i) $f(\mathbf{A}, \overline{\mathbf{R}}, \mathbf{B})=g\left(\overline{\mathbf{R}}^{T} \mathbf{A}, \overline{\mathbf{R}}^{T} \mathbf{B}\right)$;

(ii) $g$ is quasiconvex i.e.

$$
g(\mathbf{A}, \mathbf{B}) \leq \frac{1}{\operatorname{meas}(D)} \int_{D} g(\mathbf{A}+\nabla \boldsymbol{\chi}(x), \mathbf{B}+\nabla \boldsymbol{\psi}(x)) \mathrm{d} x
$$

for every open bounded set $D$ with Lipschitz boundary, for every $\mathbf{A}, \mathbf{B} \in \mathbb{R}^{3 \times m}$ and for every $\chi \in$ $W_{0}^{1, \infty}\left(D ; \mathbb{R}^{3}\right), \boldsymbol{\psi} \in W_{0}^{1, \infty}\left(D ; \mathbb{R}^{3}\right) ;$

(iii) $g$ satisfies the conditions

$|g(\mathbf{A}, \mathbf{B})| \leq K\left(1+\|\mathbf{A}\|^{p}+\|\mathbf{B}\|^{p}\right)$,

$\left|g\left(\mathbf{A}_{1}, \mathbf{B}_{1}\right)-g\left(\mathbf{A}_{2}, \mathbf{B}_{2}\right)\right| \leq K\left(1+\left\|\mathbf{A}_{1}\right\|^{p-1}+\left\|\mathbf{B}_{1}\right\|^{p-1}+\left\|\mathbf{A}_{2}\right\|^{p-1}+\left\|\mathbf{B}_{2}\right\|^{p-1}\right)\left(\left\|\mathbf{A}_{1}-\mathbf{A}_{2}\right\|+\left\|\mathbf{B}_{1}-\mathbf{B}_{2}\right\|\right)$.

Furthermore $f$ satisfies conditions (b) and (d).

If $f$ is coercive i.e. if there exist constants $C_{1}>0, C_{2}$ such that

$$
f(\mathbf{A}, \overline{\mathbf{R}}, \mathbf{B}) \geq C_{1}\left(\|\mathbf{A}\|^{p}+\|\mathbf{B}\|^{p}\right)+C_{2}, \quad \forall \mathbf{A}, \mathbf{B} \in \mathbb{R}^{3 \times m}, \overline{\mathbf{R}} \in \operatorname{SO}(3)
$$

then $g$ is also coercive with the same constants i.e.

$$
g(\mathbf{A}, \mathbf{B}) \geq C_{1}\left(\|\mathbf{A}\|^{p}+\|\mathbf{B}\|^{p}\right)+C_{2} .
$$

Proof. Since $f$ is objective we have

$$
f(\mathbf{Q A}, \mathbf{Q} \overline{\mathbf{R}}, \mathbf{Q B})=f(\mathbf{A}, \overline{\mathbf{R}}, \mathbf{B}), \quad \mathbf{Q} \in \mathrm{SO}(3) .
$$

Taking $\mathbf{Q}=\overline{\mathbf{R}}^{T}$ we can define $g$ by

$$
g(\mathbf{A}, \mathbf{B})=f(\mathbf{A}, \mathbf{I}, \mathbf{B}),
$$

where $\mathbf{I}$ is the identity matrix. That $g$ is quasiconvex directly follows from quasiconvexity of $f$ for the special case $\overline{\mathbf{R}}=\mathbf{I}$. Also, from the growth condition for $f$ we easily see that $g$ satisfies growth condition

$$
|g(\mathbf{A}, \mathbf{B})|<K\left(1+\|\mathbf{A}\|^{p}+\|\mathbf{B}\|^{p}\right)
$$


Since every quasiconvex function is convex in each variable (see [6]) using Lemma 4.8 we conclude

$$
\left|g\left(\mathbf{A}_{1}, \mathbf{B}_{1}\right)-g\left(\mathbf{A}_{2}, \mathbf{B}_{2}\right)\right| \leq K\left(1+\left\|\mathbf{A}_{1}\right\|^{p-1}+\left\|\mathbf{B}_{1}\right\|^{p-1}+\left\|\mathbf{A}_{2}\right\|^{p-1}+\left\|\mathbf{B}_{2}\right\|^{p-1}\right)\left(\left\|\mathbf{A}_{1}-\mathbf{A}_{2}\right\|+\left\|\mathbf{B}_{1}-\mathbf{B}_{2}\right\|\right) .
$$

$f$ satisfies (b) because

$$
\begin{aligned}
\left|f\left(\mathbf{A}_{1}, \overline{\mathbf{R}}, \mathbf{B}_{1}\right)-f\left(\mathbf{A}_{2}, \overline{\mathbf{R}}, \mathbf{B}_{2}\right)\right|= & \left|g\left(\overline{\mathbf{R}}^{T} \mathbf{A}_{1}, \overline{\mathbf{R}}^{T} \mathbf{B}_{1}\right)-g\left(\overline{\mathbf{R}}^{T} \mathbf{A}_{2}, \overline{\mathbf{R}}^{T} \mathbf{B}_{2}\right)\right| \\
\leq & K\left(1+\left\|\overline{\mathbf{R}}^{T} \mathbf{A}_{1}\right\|^{p-1}+\left\|\overline{\mathbf{R}}^{T} \mathbf{B}_{1}\right\|^{p-1}+\left\|\overline{\mathbf{R}}^{T} \mathbf{A}_{2}\right\|^{p-1}+\left\|\overline{\mathbf{R}}^{T} \mathbf{B}_{2}\right\|^{p-1}\right) \\
& \times\left(\left\|\overline{\mathbf{R}}^{T} \mathbf{A}_{1}-\overline{\mathbf{R}}^{T} \mathbf{A}_{2}\right\|+\left\|\overline{\mathbf{R}}^{T} \mathbf{B}_{1}-\overline{\mathbf{R}}^{T} \mathbf{B}_{2}\right\|\right) \\
\leq & K_{1}\left(1+\left\|\mathbf{A}_{1}\right\|^{p-1}+\left\|\mathbf{B}_{1}\right\|^{p-1}+\left\|\mathbf{A}_{2}\right\|^{p-1}+\left\|\mathbf{B}_{2}\right\|^{p-1}\right)\left(\left\|\mathbf{A}_{1}-\mathbf{A}_{2}\right\|+\left\|\mathbf{B}_{1}-\mathbf{B}_{2}\right\|\right) .
\end{aligned}
$$

Let us see why $f$ satisfies (d)

$$
\begin{aligned}
& \left|f\left(\mathbf{A}, \overline{\mathbf{R}}_{1}, \mathbf{B}\right)-f\left(\mathbf{A}, \overline{\mathbf{R}}_{2}, \mathbf{B}\right)\right|=\left|g\left(\overline{\mathbf{R}}_{1}^{T} \mathbf{A}, \overline{\mathbf{R}}_{1}^{T} \mathbf{B}\right)-g\left(\overline{\mathbf{R}}_{2}^{T} \mathbf{A}, \overline{\mathbf{R}}_{2}^{T} \mathbf{B}\right)\right| \\
& \quad \leq K\left(1+\left\|\overline{\mathbf{R}}_{1}^{T} \mathbf{A}\right\|^{p-1}+\left\|\overline{\mathbf{R}}_{1}^{T} \mathbf{B}\right\|^{p-1}+\left\|\overline{\mathbf{R}}_{2}^{T} \mathbf{A}\right\|^{p-1}+\left\|\overline{\mathbf{R}}_{2}^{T} \mathbf{B}\right\|^{p-1}\right)\left(\left\|\overline{\mathbf{R}}_{1}^{T} \mathbf{A}-\overline{\mathbf{R}}_{2}^{T} \mathbf{A}\right\|+\left\|\overline{\mathbf{R}}_{1}^{T} \mathbf{B}-\overline{\mathbf{R}}_{2}^{T} \mathbf{B}\right\|\right) \\
& \quad \leq K_{1}\left(1+\|\mathbf{A}\|^{p}+\|\mathbf{B}\|^{p}\right)\left\|\overline{\mathbf{R}}_{1}-\overline{\mathbf{R}}_{2}\right\| .
\end{aligned}
$$

The converse of Proposition 4.9 also holds.

Proposition 4.10. Let $g: \mathbb{R}^{3 \times m} \times \mathbb{R}^{3 \times m} \rightarrow \mathbb{R}$ be quasiconvex function which satisfies growth condition

$$
|g(\mathbf{A}, \mathbf{B})| \leq K\left(1+\|\mathbf{A}\|^{p}+\|\mathbf{B}\|^{p}\right) .
$$

Then the function $f: \mathbb{R}^{3 \times m} \times \mathrm{SO}(3) \times \mathbb{R}^{3 \times m} \rightarrow \mathbb{R}$ defined by:

$$
f(\mathbf{A}, \overline{\mathbf{R}}, \mathbf{B}):=g\left(\overline{\mathbf{R}}^{T} \mathbf{A}, \overline{\mathbf{R}}^{T} \mathbf{B}\right)
$$

is continuous, objective, quasiconvex in the first and last variable and satisfies the conditions (a), (b) and (d). If $g$ is coercive then $f$ is also coercive.

Proof. Let us see why $f$ is quasiconvex. Let $D$ be a cube, $\boldsymbol{\psi}, \boldsymbol{\chi} \in C_{0}^{\infty}\left(D, \mathbb{R}^{3}\right)$ and $\mathbf{A}, \mathbf{B} \in \mathbb{R}^{3 \times m}, \overline{\mathbf{R}} \in \mathrm{SO}(3)$. We have to prove that

$$
f(\mathbf{A}, \overline{\mathbf{R}}, \mathbf{B}) \leq \frac{1}{\operatorname{meas}(D)} \int_{D} f(\mathbf{A}+\nabla \boldsymbol{\chi}(x), \overline{\mathbf{R}}, \mathbf{B}+\nabla \boldsymbol{\psi}(x)) \mathrm{d} x .
$$

Since $g$ is quasiconvex and $\overline{\mathbf{R}}^{T} \boldsymbol{\psi}, \overline{\mathbf{R}}^{T} \chi \in C_{0}^{\infty}\left(D, \mathbb{R}^{3}\right)$ we have that

$$
\begin{aligned}
f(\mathbf{A}, \overline{\mathbf{R}}, \mathbf{B}) & =g\left(\overline{\mathbf{R}}^{T} \mathbf{A}, \overline{\mathbf{R}}^{T} \mathbf{B}\right) \\
& \leq \frac{1}{\operatorname{meas}(D)} \int_{D} g\left(\overline{\mathbf{R}}^{T} \mathbf{A}+\overline{\mathbf{R}}^{T} \nabla \boldsymbol{\chi}(x), \overline{\mathbf{R}}^{T} \mathbf{B}+\overline{\mathbf{R}}^{T} \nabla \boldsymbol{\psi}(x)\right) \mathrm{d} x \\
& =\frac{1}{\operatorname{meas}(D)} \int_{D} f(\mathbf{A}+\nabla \boldsymbol{\chi}(x), \overline{\mathbf{R}}, \mathbf{B}+\nabla \boldsymbol{\psi}(x)) \mathrm{d} x .
\end{aligned}
$$

The objectivity of $f$ follows immediately. The continuity of $f$ is the direct consequence of the continuity of $g$. The condition (a) is the direct consequence of the $p$-growth of $g$. The conditions (b) and (d) follow in the same way as in Proposition 4.9 using the fact that, since it is quasiconvex, $g$ satisfies

$$
\left|g\left(\mathbf{A}_{1}, \mathbf{B}_{1}\right)-g\left(\mathbf{A}_{2}, \mathbf{B}_{2}\right)\right| \leq K\left(1+\left\|\mathbf{A}_{1}\right\|^{p-1}+\left\|\mathbf{B}_{1}\right\|^{p-1}+\left\|\mathbf{A}_{2}\right\|^{p-1}+\left\|\mathbf{B}_{2}\right\|^{p-1}\right)\left(\left\|\mathbf{A}_{1}-\mathbf{A}_{2}\right\|+\left\|\mathbf{B}_{1}-\mathbf{B}_{2}\right\|\right) .
$$


To prove the coerciveness of $f$ if $g$ is coercive let us note that an arbitrary norm $\|\cdot\|$ is equivalent with some unitary invariant norm of matrices. Therefore there exists a constant $C>0$ such that

$$
\|\overline{\mathbf{R}} \mathbf{A}\| \geq C\|\mathbf{A}\|, \quad \mathbf{A} \in \mathbb{R}^{3 \times m}, \overline{\mathbf{R}} \in \mathrm{SO}(3) .
$$

The coerciveness of $f$ is now a direct consequence of the coerciveness of $g$.

Finally, we restate the Theorem 4.6 for objective function $f$.

Corollary 4.11. Let $\Omega \subset \mathbb{R}^{m}$ be an open bounded set with the Lipschitz boundary and $m<p<\infty$. Let $f: \mathbb{R}^{3 \times m} \times \mathrm{SO}(3) \times \mathbb{R}^{3 \times m} \rightarrow \mathbb{R}$ be a quasiconvex in the first and the last variable, objective function which satisfies (a) and (c). Let

$$
I(\boldsymbol{\varphi}, \overline{\mathbf{R}})=\int_{\Omega} f(\nabla \boldsymbol{\varphi}(x), \overline{\mathbf{R}}(x), \boldsymbol{\omega}(x)) \mathrm{d} x .
$$

Then for every sequence $\left(\left(\varphi^{k}, \overline{\mathbf{R}}^{k}\right)\right)_{k} \subset W^{1, p}\left(\Omega, \mathbb{R}^{3}\right) \times W^{1, p}(\Omega, \mathrm{SO}(3))$ which converges weakly to $(\boldsymbol{\varphi}, \overline{\mathbf{R}})$ in $W^{1, p}\left(\Omega, \mathbb{R}^{3}\right) \times W^{1, p}\left(\Omega, \mathbb{R}^{3 \times 3}\right)$ one has

$$
I(\varphi, \overline{\mathbf{R}}) \leq \liminf _{k} I\left(\varphi^{k}, \overline{\mathbf{R}}^{k}\right)
$$

\section{REFERENCES}

[1] I. Aganović, J. Tambača and Z. Tutek, Derivation and justification of the models of rods and plates from linearized threedimensional micropolar elasticity. J. Elasticity 84 (2006) 131-152.

[2] A. Ambrosetti and G. Prodi, A Primer of Nonlinear Analysis. Cambridge University Press, Cambridge (1993).

[3] J.M. Ball, Convexity conditions and existence theorems in nonlinear elasticity. Arch. Rational Mech. Anal. 63 (1976/1977) 337-403.

[4] P.G. Ciarlet, Mathematical elasticity - Volume I: Three-dimensional elasticity. North-Holland Publishing Co., Amsterdam (1988).

[5] E. Cosserat and F. Cosserat, Théorie des corps déformables. Librairie Scientifique A. Hermann et Fils [Theory of deformable bodies], Paris (1909).

[6] B. Dacorogna, Direct methods in the calculus of variations. Springer-Verlag, Berlin (1989).

[7] A.C. Eringen, Microcontinuum Field Theories - Volume 1: Foundations and Solids. Springer-Verlag, New York (1999).

[8] G.B. Folland, Real analysis, Modern techniques and their applications. John Wiley \& Sons, Inc., New York (1984).

[9] I. Hlaváček and M. Hlaváček, On the existence and uniqueness of solution and some variational principles in linear theories of elasticity with couple-stresses. I. Cosserat continuum. Appl. Math. 14 (1969) 387-410.

[10] J. Jeong and P. Neff, Existence, uniqueness and stability in linear Cosserat elasticity for weakest curvature conditions. Math. Mech. Solids (2008) DOI: 10.1177/1081286508093581. Preprint 2550 available at http://www3.mathematik.tu-darmstadt.de/ $\mathrm{fb} / \mathrm{mathe/bibliothek/preprints.html.}$

[11] P.M. Mariano and G. Modica, Ground states in complex bodies. ESAIM: COCV (2008) published online, DOI: 10.1051/cocv:2008036.

[12] N.G. Meyers, Quasi-convexity and lower semi-continuity of multiple variational integrals of any order. Trans. Amer. Math. Soc. 119 (1965) 125-149.

[13] P. Neff, On Korn's first inequality with nonconstant coefficients. Proc. R. Soc. Edinb. Sect. A 132 (2002) 221-243.

[14] P. Neff, Existence of minimizers for a geometrically exact Cosserat solid. Proc. Appl. Math. Mech. 4 (2004) 548-549.

[15] P. Neff, A geometrically exact Cosserat-shell model including size effects, avoiding degeneracy in the thin shell limit, Part I: Formal dimensional reduction for elastic plates and existence of minimizers for positive Cosserat couple modulus. Cont. Mech. Thermodynamics 16 (2004) 577-628.

[16] P. Neff, The Cosserat couple modulus for continuous solids is zero viz the linearized Cauchy-stress tensor is symmetric. $Z$. Angew. Math. Mech. 86 (2006) 892-912. Preprint 2409 available at http://www3.mathematik.tu-darmstadt.de/fb/mathe/ bibliothek/preprints.html.

[17] P. Neff, Existence of minimizers for a finite-strain micromorphic elastic solid. Proc. Roy. Soc. Edinb. A 136 (2006) $997-1012$. Preprint 2318 available at http://wwwbib.mathematik.tu-darmstadt.de/Math-Net/Preprints/Listen/pp04.html.

[18] P. Neff, A finite-strain elastic-plastic Cosserat theory for polycrystals with grain rotations. Int. J. Eng. Sci. 44 (2006) 574-594.

[19] P. Neff, A geometrically exact planar Cosserat shell-model with microstructure. Existence of minimizers for zero Cosserat couple modulus. Math. Meth. Appl. Sci. 17 (2007) 363-392. Preprint 2357 available at http://www3.mathematik.tu-darmstadt.de/ $\mathrm{fb} /$ mathe/bibliothek/preprints.html. 
[20] P. Neff and K. Chelminski, A geometrically exact Cosserat shell-model for defective elastic crystals. Justification via $\Gamma$-convergence. Interfaces Free Boundaries 9 (2007) 455-492.

[21] P. Neff and S. Forest, A geometrically exact micromorphic model for elastic metallic foams accounting for affine microstructure. Modelling, existence of minimizers, identification of moduli and computational results. J. Elasticity 87 (2007) 239-276.

[22] P. Neff and I. Münch, Curl bounds Grad on SO(3). ESAIM: COCV 14 (2008) 148-159. Preprint 2455 available at http:// www3.mathematik.tu-darmstadt.de/fb/mathe/bibliothek/preprints.html.

[23] W. Nowacki, Theory of asymmetric elasticity. Oxford, Pergamon (1986).

$[24]$ W. Pompe, Korn's first inequality with variable coefficients and its generalizations. Commentat. Math. Univ. Carolinae 44 (2003) 57-70.

[25] J. Tambača and I. Velčić, Derivation of a model of nonlinear micropolar plate. (Submitted). 